\title{
Metal-Catalyzed Cotrimerization of Arynes and Alkenes
}

lago Quintana, Arnold J. Boersma, ${ }^{\dagger}$ Diego Peña, ${ }^{*}$ Dolores Pérez, ${ }^{\star}$ and Enrique Guitián Departamento de Química Orgánica, Universidad de Santiago de Compostela,

15782 Santiago de Compostela, Spain

qodpena@usc.es,qodolopm@usc.es

Org. Lett.

\section{SUPPORTING INFORMATION}

\section{CONTENTS}

Experimental details:

General procedure for the metal-catalyzed cotrimerization of arynes and alkenes

Spectroscopic data:

Dihydrophenanthrenes (3a-f and 6a,b) and $o$-olefinated biaryls (4a-f and 7a,b)

Experiment with deuterium-labeled methyl acrylate $\left(\beta, \beta-d_{2}-\mathbf{2 a}\right)$

Page S8

Experiment using regioisomeric triflates 13a,b

Page S10

${ }^{1} \mathrm{H}$ and ${ }^{13} \mathrm{C}$ NMR spectra

Page S11

\footnotetext{
${ }^{\dagger}$ Present address: Department of Organic and Molecular Inorganic Chemistry, University of Groningen, Nijenborgh 4, 9747AG, Groningen, The Netherlands
} 


\section{Experimental details}

All reactions were performed under argon atmosphere using oven-dried glassware and standard Schlenk techniques. Solvents were dried by distillation from a drying agent before use: $\mathrm{MeCN}$ from $\mathrm{CaH}_{2}$ and toluene from $\mathrm{Na}$.

TLC was performed on Merck silica gel $60 \mathrm{~F}_{254}$; chromatograms were visualized with UV light (254 and $360 \mathrm{~nm}$ ). Flash column chromatography was performed on Merck silica gel 60 (ASTM 230-400 mesh). ${ }^{1} \mathrm{H}$ and ${ }^{13} \mathrm{C}$ NMR spectra were recorded at 250.13 and $62.83 \mathrm{MHz}$ (Bruker DPX-250 instrument), 300 and $75 \mathrm{MHz}$ (Varian Mercury-300 instrument) or 400 and $100 \mathrm{MHz}$ (Varian Mercury-400 instrument), respectively. Low-resolution electron impact mass spectra (EI-LRMS) were determined at $70 \mathrm{eV}$ on a HP-5988A instrument. High-resolution mass spectra (HRMS) were obtained on a Micromass Autospec spectrometer. Melting points were measured on a Gallenkamp instrument. IR spectra were recorded on a Mattson Cygnus 100 spectrophotometer. GC/MS analyses were performed on a Agilent 6890N/5973inert instrument with a HP-5MS capillary column.

Triflates $\mathbf{1},{ }^{1} \mathbf{5 a},{ }^{2} \mathbf{5 b},{ }^{3} \mathbf{1 3} \mathbf{a}^{1 \mathrm{~b}}$ and $\mathbf{1 3 b},{ }^{4} \mathrm{Pd}\left(\mathrm{PPh}_{3}\right)_{4}{ }^{5}$ and deuterium-labeled methyl acrylate $\left(\beta, \beta-d_{2}-2 \mathbf{a}\right)^{6}$ were prepared following published procedures. Commercial reagents were purchased from ABCR GmbH, Aldrich Chemical Co., or Strem Chemicals Inc., and were used without further purification.

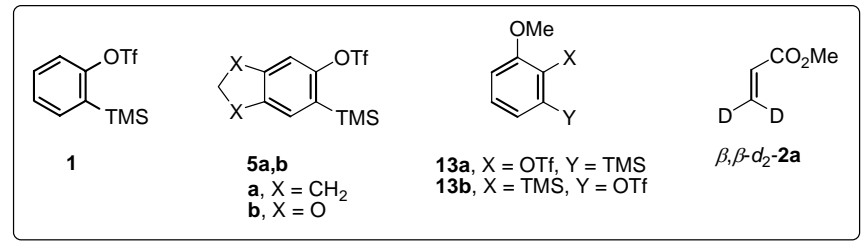

Figure S1

\section{General procedure for the metal-catalyzed cotrimerization of arynes and alkenes}

The aryne precursor $(\mathbf{1}, \mathbf{5 a}, \mathbf{b}$ or 13a,b, $0.5 \mathrm{mmol}$ aprox.) was added to a suspension of finely powdered anhydrous CsF ( 2 equiv), the corresponding alkene (2a-f, 3 equiv) and the metal catalyst ( 0.1 equiv) in 1:1 MeCN/toluene $(5 \mathrm{~mL})$. The mixture was stirred under argon at $60{ }^{\circ} \mathrm{C}$ for $16 \mathrm{~h}$. The solvent was evaporated and the residue was purified by column chromatography to isolate dihydrophenanthrenes (3a-f or 6a,b) and $o$-olefinated biaryls (4a-f or 7a,b).

In all the experiments dihydrophenanthrenes and o-olefinated biaryls were first isolated as a mixture, in order to determine the combined yield and the mole ratio ( ${ }^{1} \mathrm{H}$ NMR). Further careful separation of these products by column chromatography led to pure samples for spectroscopic characterization.
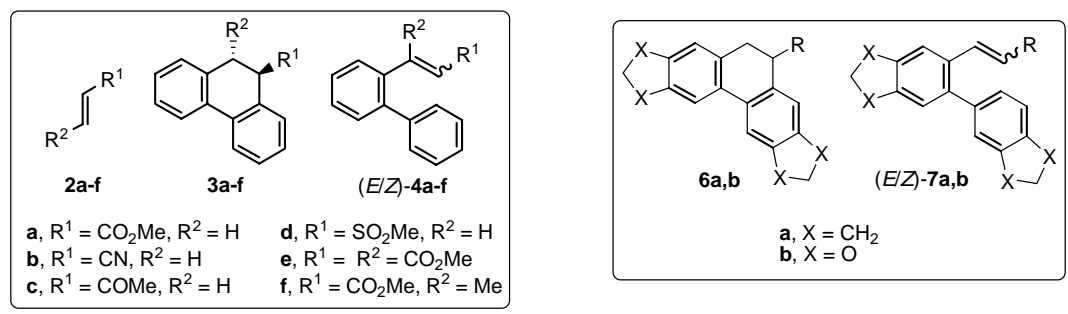

Figure S2

The solvent mixture (1:1 MeCN/toluene) had a crucial influence in the ratio of products (3:4). For instance, the higher was the proportion of $\mathrm{MeCN}$, the higher was the selectivity in favour of 3 . However, the use of a more polar solvent mixture increases the rate of benzyne generation, so its cyclotrimerization product (triphenylene) was isolated in undesirable notable amounts.

\footnotetext{
1 (a) Himeshima, Y.; Sonoda, T.; Kobayashi, H. Chem. Lett. 1983, 1211. (b) Peña, D.; Cobas, A.; Pérez, D.; Guitián, E. Synthesis 2002, 1454.

Yoshida, H.; Ikadai, J.; Shudo, M.; Ohshita, J.; Kunai, A. J. Am. Chem. Soc. 2003, 125, 6638.

4 Sato, Y.; Tamura, T.; Mori, M. Angew. Chem. Int. Ed. 2004, 43, 2436.

Peña, D.; Pérez, D.; Guitián, E.; Castedo, L. J. Am. Chem. Soc. 1999, 121, 5827.

Hegedus, L. S. Palladium in Organic Synthesis. In Organometallics in Synthesis: A Manual; Schlosser, M., Ed.; John Wiley \& Sons: New York, 1994.

${ }^{6}$ Meltzer, A. D.; Tirrell, D. A.; Jones, A. A.; Inglefield, P. T. Macromolecules 1992, 25, 4549.
} 
The cotrimerization of benzyne and acrylonitrile (2b) was particularly interesting. Using an excess of alkene (6 equiv) in $\mathrm{MeCN}, 3 \mathbf{b}$ evolved in situ in a tandem $\alpha \mathrm{C}-\mathrm{H}$ activation-Michael addition to acrylonitrile (Scheme S1). Consequently, compound 17 was isolated by a cascade sequence, resulting from the overall reaction of two benzynes and two alkenes, in a one pot process with the formation of four $\mathrm{C}-\mathrm{C}$ bonds. Apparently, the $\alpha \mathrm{C}-\mathrm{H}$ activation of $\mathbf{3 b}$ is promoted by $\mathrm{CsF}$, since in the absence of palladium catalyst $\mathbf{3 b}$ also evolved to afford compound $\mathbf{1 7}$.

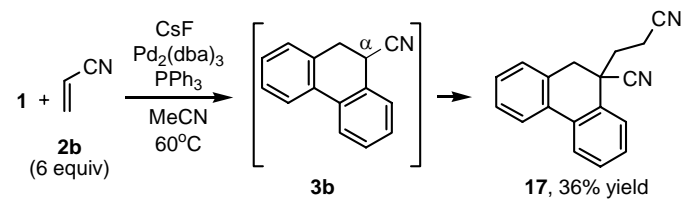

Scheme S1 


\section{Spectroscopic data}

Methyl 9,10-dihydro-9-phenanthrenecarboxylate (3a) ${ }^{7}$

Column chromatography $\left(\mathrm{SiO}_{2} ; 1: 15 \mathrm{AcOEt} /\right.$ hexane) yielded 3a as a yellow oil. ${ }^{1} \mathrm{H}$ NMR $(300 \mathrm{MHz}$, $\left.\mathrm{CDCl}_{3}\right) \delta=7.78(\mathrm{~d}, J=7.9 \mathrm{~Hz}, 1 \mathrm{H}), 7.74(\mathrm{~d}, J=7.7 \mathrm{~Hz}, 1 \mathrm{H}), 7.43-7.24(\mathrm{~m}, 6 \mathrm{H}), 3.89(\mathrm{t}, J=5.8 \mathrm{~Hz}, 1 \mathrm{H})$, $3.68(\mathrm{~s}, 3 \mathrm{H}), 3.32(\mathrm{dd}, J=15.3,5.9 \mathrm{~Hz}, 1 \mathrm{H}), 3.12(\mathrm{dd}, J=15.3,5.8 \mathrm{~Hz}, 1 \mathrm{H}) \mathrm{ppm} ;{ }^{13} \mathrm{C}$ NMR $(75 \mathrm{MHz}$, $\left.\mathrm{CDCl}_{3}\right) \delta=173.5(\mathrm{C}), 134.5(\mathrm{C}), 134.1(\mathrm{C}), 133.8(\mathrm{C}), 133.6(\mathrm{C}), 128.6(\mathrm{CH}), 128.4(\mathrm{CH}), 128.1(\mathrm{CH})$, $127.8(\mathrm{CH}), 127.6(\mathrm{CH}), 127.3(\mathrm{CH}), 124.0(\mathrm{CH}), 123.7(\mathrm{CH}), 52.1\left(\mathrm{CH}_{3}\right), 44.6(\mathrm{CH}), 31.7\left(\mathrm{CH}_{2}\right) \mathrm{ppm}$; MS (EI), m/z (\%): 238 (14), 179 (100); HRMS (EI) for $\mathrm{C}_{16} \mathrm{H}_{14} \mathrm{O}_{2}$, calcd: 238.0994, found: 238.0995; IR (CsI): $1735 \mathrm{~cm}^{-1}$.

Methyl (E)-3-(1,1'-biphenyl-2-yl)-2-propenoate [(E)-4a]

Column chromatography $\left(\mathrm{SiO}_{2} ; 1: 15\right.$ AcOEt/hexane) yielded $(E)-\mathbf{4 a}$ as a colourless oil. ${ }^{1} \mathrm{H}$ NMR $(300$ $\left.\mathrm{MHz}, \mathrm{CDCl}_{3}\right) \delta=7.80-7.65(\mathrm{~m}, 2 \mathrm{H}), 7.49-7.23(\mathrm{~m}, 8 \mathrm{H}), 6.40(\mathrm{~d}, \mathrm{~J}=15.9 \mathrm{~Hz}, 1 \mathrm{H}), 3.74(\mathrm{~s}, 3 \mathrm{H}) \mathrm{ppm} ;{ }^{13} \mathrm{C}$ NMR (75 MHz, $\left.\mathrm{CDCl}_{3}\right) \delta=167.3(\mathrm{C}), 143.9(\mathrm{CH}), 142.9(\mathrm{C}), 139.8(\mathrm{C}), 132.5(\mathrm{C}), 130.5(\mathrm{CH}), 129.85$ $(\mathrm{CH}), 129.75(2 \mathrm{CH}), 128.3(2 \mathrm{CH}), 127.6(\mathrm{CH}), 127.5(\mathrm{CH}), 126.8(\mathrm{CH}), 118.7(\mathrm{CH}), 51.6\left(\mathrm{CH}_{3}\right) \mathrm{ppm}$; MS (EI), $m / z$ (\%): 238 (16), 179 (100); HRMS (EI) for $\mathrm{C}_{16} \mathrm{H}_{14} \mathrm{O}_{2}$, calcd: 238.0994, found: 238.0989.

Methyl (Z)-3-(1,1'-biphenyl-2-yl)-2-propenoate [(Z)-4a]

Column chromatography $\left(\mathrm{SiO}_{2} ; 1: 15 \mathrm{AcOEt} /\right.$ hexane) yielded (Z)-4a as a colourless oil. ${ }^{1} \mathrm{H}$ NMR (300 $\left.\mathrm{MHz}, \mathrm{CDCl}_{3}\right) \delta=7.59-7.51(\mathrm{~m}, 2 \mathrm{H}), 7.45-7.31(\mathrm{~m}, 7 \mathrm{H}), 6.85(\mathrm{~d}, J=12.2 \mathrm{~Hz}, 1 \mathrm{H}), 5.93(\mathrm{~d}, J=12.2 \mathrm{~Hz}$, $1 \mathrm{H}), 3.69(\mathrm{~s}, 3 \mathrm{H}) \mathrm{ppm} ;{ }^{13} \mathrm{C}$ NMR $\left(75 \mathrm{MHz}, \mathrm{CDCl}_{3}\right) \delta=166.8(\mathrm{C}), 144.3(\mathrm{CH}), 141.3(\mathrm{C}), 140.5(\mathrm{C})$, $133.6(\mathrm{C}), 129.9(\mathrm{CH}), 129.8(2 \mathrm{CH}), 129.4(\mathrm{CH}), 128.9(\mathrm{CH}), 128.1(2 \mathrm{CH}), 127.4(\mathrm{CH}), 126.7(\mathrm{CH})$, $119.8(\mathrm{CH}), 51.3\left(\mathrm{CH}_{3}\right) \mathrm{ppm}$; MS (EI), m/z (\%): 238 (15), 179 (100); HRMS (EI) for $\mathrm{C}_{16} \mathrm{H}_{14} \mathrm{O}_{2}$, calcd: 238.0994, found: 238.0984 .

\section{9,10-Dihydro-9-phenanthrenecarbonitrile (3b) $)^{7,8}$}

Column chromatography $\left(\mathrm{SiO}_{2}, 1: 50 \mathrm{AcOEt} /\right.$ hexane) yielded $3 \mathbf{b}$ as a yellow oil. ${ }^{1} \mathrm{H}$ NMR $(400 \mathrm{MHz}$, $\left.\mathrm{CDCl}_{3}\right) \delta=7.82-7.27(\mathrm{~m}, 8 \mathrm{H}), 4.08(\mathrm{t}, J=7.8 \mathrm{~Hz}, 1 \mathrm{H}), 3.21(\mathrm{~d}, J=7.9 \mathrm{~Hz}, 2 \mathrm{H}) \mathrm{ppm} ; \mathrm{MS}(\mathrm{EI}), \mathrm{m} / \mathrm{z}(\%)$ : 205 (100), 190 (22).

\section{(E)-3-(1,1'-Biphenyl-2-yl)-2-propenenitrile [(E)-4b]}

Column chromatography $\left(\mathrm{SiO}_{2} ; 1: 50 \mathrm{AcOEt} /\right.$ hexane $)$ yielded $(E)-\mathbf{4 b}$ as a colourless oil. ${ }^{1} \mathrm{H}$ NMR $(300$ $\left.\mathrm{MHz}, \mathrm{CDCl}_{3}\right) \delta=7.62(\mathrm{~d}, J=7.8 \mathrm{~Hz}, 1 \mathrm{H}), 7.53-7.25(\mathrm{~m}, 9 \mathrm{H}), 5.85(\mathrm{~d}, J=16.6 \mathrm{~Hz}, 1 \mathrm{H}) \mathrm{ppm} ;{ }^{13} \mathrm{C}$ NMR $\left(75 \mathrm{MHz}, \mathrm{CDCl}_{3}\right) \delta=149.7(\mathrm{CH}), 142.7(\mathrm{C}), 139.2(\mathrm{C}), 131.6(\mathrm{C}), 130.8(\mathrm{CH}), 130.7(\mathrm{CH}), 129.6(2 \mathrm{CH})$, $128.5(2 \mathrm{CH}), 127.9(\mathrm{CH}), 127.8(\mathrm{CH}), 125.8(\mathrm{CH}), 118.2(\mathrm{C}), 96.8(\mathrm{CH}) \mathrm{ppm} ; \mathrm{MS}(\mathrm{EI}), \mathrm{m} / \mathrm{z}(\%): 205$ (100), 190 (35); HRMS (EI) for $\mathrm{C}_{15} \mathrm{H}_{11} \mathrm{~N}$, calcd: 205.0891, found: 205.0884 .

(Z)-3-(1,1'-Biphenyl-2-yl)-2-propenenitrile [(Z)-4b]

Column chromatography $\left(\mathrm{SiO}_{2} ; 1: 50 \mathrm{AcOEt} /\right.$ hexane) yielded $(\mathrm{Z})-\mathbf{4 b}$ as a colourless oil. ${ }^{1} \mathrm{H}$ NMR (300 $\left.\mathrm{MHz}, \mathrm{CDCl}_{3}\right) \delta=8.15(\mathrm{~m}, 1 \mathrm{H}), 7.61-7.19(\mathrm{~m}, 8 \mathrm{H}), 7.11(\mathrm{~d}, J=12.1 \mathrm{~Hz}, 1 \mathrm{H}), 5.42(\mathrm{~d}, J=12.0 \mathrm{~Hz}, 1 \mathrm{H})$ ppm; ${ }^{13} \mathrm{C}$ NMR $\left(75 \mathrm{MHz}, \mathrm{CDCl}_{3}\right) \delta=148.7(\mathrm{CH}), 142.6(\mathrm{C}), 139.6(\mathrm{C}), 131.6(\mathrm{C}), 130.5(\mathrm{CH}), 130.2$ (CH), $129.8(2 \mathrm{CH}), 128.3(2 \mathrm{CH}), 128.0(\mathrm{CH}), 127.7(2 \mathrm{CH}), 117.3(\mathrm{C}), 96.1(\mathrm{CH}) ; \mathrm{MS}(\mathrm{EI}), \mathrm{m} / \mathrm{z}(\%): 205$ (100), 190 (41); HRMS (EI) for $\mathrm{C}_{15} \mathrm{H}_{11} \mathrm{~N}$, calcd: 205.0891, found: 205.0896.

\section{9-Acetyl-9,10-dihydrophenanthrene (3c) ${ }^{7}$}

Column chromatography $\left(\mathrm{SiO}_{2} ; 1: 40 \mathrm{AcOEt} /\right.$ hexane) yielded $3 \mathrm{c}$ as a light brown oil. ${ }^{1} \mathrm{H} \mathrm{NMR}(400 \mathrm{MHz}$, $\left.\mathrm{CDCl}_{3}\right) \delta=7.79(\mathrm{~d}, J=7.6 \mathrm{~Hz}, 1 \mathrm{H}), 7.73(\mathrm{~d}, J=7.7 \mathrm{~Hz}, 1 \mathrm{H}), 7.42-7.21(\mathrm{~m}, 6 \mathrm{H}), 3.73(\mathrm{dd}, J=6.1,3.6$ $\mathrm{Hz}, 1 \mathrm{H}), 3.32(\mathrm{dd}, J=15.4,3.6 \mathrm{~Hz}, 1 \mathrm{H}), 3.13(\mathrm{dd}, J=15.4,6.1 \mathrm{~Hz}, 1 \mathrm{H}), 1.96(\mathrm{~s}, 3 \mathrm{H}) \mathrm{ppm} ;{ }^{13} \mathrm{C} \mathrm{NMR}$ $\left(100 \mathrm{MHz}, \mathrm{CDCl}_{3}\right) \delta=208.6(\mathrm{C}), 134.7(\mathrm{C}), 134.4(\mathrm{C}), 134.3(\mathrm{C}), 133.4(\mathrm{C}), 129.2(\mathrm{CH}), 128.6(\mathrm{CH})$, $128.2(\mathrm{CH}), 127.9(\mathrm{CH}), 127.7(\mathrm{CH}), 127.3(\mathrm{CH}), 124.3(\mathrm{CH}), 123.6(\mathrm{CH}), 52.6(\mathrm{CH}), 31.2\left(\mathrm{CH}_{2}\right), 28.6$ $\left(\mathrm{CH}_{3}\right) \mathrm{ppm}$; MS (EI), m/z (\%): 222 (11), 179 (100); HRMS (ESI) for $\mathrm{C}_{16} \mathrm{H}_{14} \mathrm{ONa}\left(\mathrm{M}^{+}+\mathrm{Na}\right)$, calcd: 245.0943, found: 245.0940; IR (CsI): $1710 \mathrm{~cm}^{-1}$.

(E)-4-(1,1'-Biphenyl-2-yl)-3-buten-2-one [(E)-4c]

Column chromatography $\left(\mathrm{SiO}_{2} ; 1: 40 \mathrm{AcOEt} /\right.$ hexane) yielded $(E)-4 \mathrm{c}$ as a yellow oil. ${ }^{1} \mathrm{H}$ NMR $(300 \mathrm{MHz}$, $\left.\mathrm{CDCl}_{3}\right) \delta=7.75-7.22(\mathrm{~m}, 10 \mathrm{H}), 6.65(\mathrm{~d}, J=16.3 \mathrm{~Hz}, 1 \mathrm{H}), 2.23(\mathrm{~s}, 3 \mathrm{H}) \mathrm{ppm} ;{ }^{13} \mathrm{C}$ NMR $\left(75 \mathrm{MHz}, \mathrm{CDCl}_{3}\right)$

\footnotetext{
${ }_{8}^{7}$ Harvey, R. G.; Fu, P. P.; Rabideau, P. W. J. Org. Chem. 1976, 41, 3722.

8 Lewis, F. D.; Zebrowski, B. E.; Correa, P. E. J. Am. Chem. Soc. 1984, 106, 187.
} 
$\delta=198.6(\mathrm{C}), 142.9(\mathrm{CH}), 139.8(\mathrm{C}), 132.5(\mathrm{C}), 130.4(\mathrm{CH}), 130.0(\mathrm{CH}), 129.8(2 \mathrm{CH}), 128.9(\mathrm{C}), 128.4$ $(\mathrm{CH}), 128.2(2 \mathrm{CH}), 127.7(\mathrm{CH}), 127.6(\mathrm{CH}), 126.7(\mathrm{CH}), 26.9\left(\mathrm{CH}_{3}\right) \mathrm{ppm} ; \mathrm{MS}(\mathrm{EI}), \mathrm{m} / \mathrm{z}(\%): 222(2)$, 179 (100); HRMS (EI) for $\mathrm{C}_{16} \mathrm{H}_{14} \mathrm{O}$, calcd: 222.1045 , found: 222.1045.

(Z)-4-(1,1'-Biphenyl-2-yl)-3-buten-2-one [(Z)-4c]

Column chromatography $\left(\mathrm{SiO}_{2} ; 1: 40 \mathrm{AcOEt} /\right.$ hexane) yielded $(\mathrm{Z})-4 \mathrm{c}$ as a yellow oil. ${ }^{1} \mathrm{H}$ NMR $(300 \mathrm{MHz}$, $\left.\mathrm{CDCl}_{3}\right) \delta=7.71-7.20(\mathrm{~m}, 9 \mathrm{H}), 6.86(\mathrm{~d}, J=12.3 \mathrm{~Hz}, 1 \mathrm{H}), 6.12(\mathrm{~d}, J=12.3 \mathrm{~Hz}, 1 \mathrm{H}), 2.14(\mathrm{~s}, 3 \mathrm{H}) \mathrm{ppm}$; ${ }^{13} \mathrm{C}$ NMR $\left(75 \mathrm{MHz}, \mathrm{CDCl}_{3}\right) \delta=201.1(\mathrm{C}), 141.1(\mathrm{CH}), 140.3(\mathrm{C}), 134.0(\mathrm{C}), 130.2(\mathrm{CH}), 129.8(\mathrm{CH})$, $129.75(\mathrm{CH}), 129.6(2 \mathrm{CH}), 129.1(\mathrm{CH}), 128.8(\mathrm{C}), 128.1(2 \mathrm{CH}), 127.4(\mathrm{CH}), 127.1(\mathrm{CH}), 30.5\left(\mathrm{CH}_{3}\right)$ ppm; MS (EI), m/z (\%): 222(3), 179 (100); HRMS (EI) for $\mathrm{C}_{16} \mathrm{H}_{14} \mathrm{O}$, calcd: 222.1045, found: 222.1041.

\section{9-(Methylsulfonyl)-9,10-dihydrophenanthrene (3d)}

Column chromatography $\left(\mathrm{SiO}_{2} ; 1: 15\right.$ AcOEt/hexane) yielded 3d as a dark yellow oil. ${ }^{1} \mathrm{H}$ NMR (400 $\left.\mathrm{MHz} \mathrm{CDCl}_{3}\right) \delta=7.87(\mathrm{~d}, J=7.9 \mathrm{~Hz}, 1 \mathrm{H}), 7.79(\mathrm{~d}, J=7.8 \mathrm{~Hz}, 1 \mathrm{H}), 7.57(\mathrm{~d}, J=7.7 \mathrm{~Hz}, 1 \mathrm{H}), 7.49(\mathrm{t}, J=$ $8.3 \mathrm{~Hz}, 1 \mathrm{H}), 7.43-7.22(\mathrm{~m}, 4 \mathrm{H}), 4.25(\mathrm{~d}, J=6.9 \mathrm{~Hz}, 1 \mathrm{H}), 3.72(\mathrm{dd}, J=17.0,1.4 \mathrm{~Hz}, 1 \mathrm{H}), 3.50(\mathrm{dd}, J=$ 17.0, 7.0 Hz, 1H), 2.06 (s, 3H) ppm; $\left.{ }^{13} \mathrm{C} \mathrm{NMR} \mathrm{(100} \mathrm{MHz,} \mathrm{CDCl}_{3}\right) \delta=134.3(\mathrm{C}), 132.9(\mathrm{C}), 131.9(\mathrm{CH})$, $131.5(\mathrm{C}), 130.2(\mathrm{CH}), 129.0(\mathrm{CH}), 128.8(\mathrm{CH}), 128.3(\mathrm{CH}), 128.2(\mathrm{CH}), 127.7(\mathrm{C}), 124.1(\mathrm{CH}), 123.8$ $(\mathrm{CH}), 64.6(\mathrm{CH}), 37.7\left(\mathrm{CH}_{3}\right), 29.4\left(\mathrm{CH}_{2}\right) \mathrm{ppm}$; MS (EI), m/z (\%): $178\left(\mathrm{M}^{+}-\mathrm{HSO}_{2} \mathrm{Me}, 100\right)$; HRMS (EI) for $\mathrm{C}_{14} \mathrm{H}_{10}\left(\mathrm{M}^{+}-\mathrm{HSO}_{2} \mathrm{Me}\right)$, calcd: 178.0782, found: 178.0779; IR (CsI): $1302,1118 \mathrm{~cm}^{-1}$.

\section{2-[(E)-2-(Methylsulfonyl)-1-ethenyl]-1,1'-biphenyl [(E)-4d]}

Column chromatography $\left(\mathrm{SiO}_{2} ; 1: 15 \mathrm{AcOEt} /\right.$ hexane) yielded $(E)-\mathbf{4 d}$ as a colourless oil. ${ }^{1} \mathrm{H}$ NMR $(300$ $\left.\mathrm{MHz}, \mathrm{CDCl}_{3}\right) \delta=7.72-7.61(\mathrm{~m}, 2 \mathrm{H}), 7.55-7.37(\mathrm{~m}, 6 \mathrm{H}), 7.33-7.24(\mathrm{~m}, 2 \mathrm{H}), 6.81(\mathrm{~d}, J=15.5 \mathrm{~Hz}, 1 \mathrm{H})$, $2.96(\mathrm{~s}, 3 \mathrm{H}) \mathrm{ppm} ;{ }^{13} \mathrm{C} \mathrm{NMR}\left(75 \mathrm{MHz}, \mathrm{CDCl}_{3}\right) \delta=143.5(\mathrm{C}), 143.1(\mathrm{CH}), 139.2(\mathrm{C}), 130.9(\mathrm{CH}), 130.8$ $(\mathrm{CH}), 130.1(\mathrm{C}), 129.6(2 \mathrm{CH}), 128.5(2 \mathrm{CH}), 127.9(\mathrm{CH}), 127.8(\mathrm{CH}), 127.3(\mathrm{CH}), 127.2(\mathrm{CH}), 43.1$ $\left(\mathrm{CH}_{3}\right) \mathrm{ppm}$; MS (EI), m/z (\%): $178\left(\mathrm{M}^{+}-\mathrm{HSO}_{2} \mathrm{Me}, 100\right)$; HRMS (EI) for $\mathrm{C}_{14} \mathrm{H}_{10}\left(\mathrm{M}^{+}-\mathrm{HSO}_{2} \mathrm{Me}\right)$, calcd: 178.0782, found: 178.0781 .

\section{2-[(Z)-2-(Methylsulfonyl)-1-ethenyl]-1,1'-biphenyl [(Z)-4d]}

Column chromatography $\left(\mathrm{SiO}_{2} ; 1: 15 \mathrm{AcOEt} /\right.$ hexane) yielded $(Z)-4 d$ as a colourless oil. ${ }^{1} \mathrm{H}$ NMR $(250$ $\left.\mathrm{MHz}, \mathrm{CDCl}_{3}\right) \delta=7.63-7.28(\mathrm{~m}, 9 \mathrm{H}), 7.03(\mathrm{~d}, J=12.0 \mathrm{~Hz}, 1 \mathrm{H}), 6.42(\mathrm{~d}, J=12.0 \mathrm{~Hz}, 1 \mathrm{H}), 2.91(\mathrm{~s}, 3 \mathrm{H})$ ppm; ${ }^{13} \mathrm{C}$ NMR $\left(75 \mathrm{MHz}, \mathrm{CDCl}_{3}\right) \delta=141.9(\mathrm{CH}), 141.7(\mathrm{C}), 139.8(\mathrm{C}), 130.9(\mathrm{CH}), 130.8(\mathrm{C}), 130.3$ $(\mathrm{CH}), 130.1(\mathrm{CH}), 129.7(\mathrm{CH}), 129.5(2 \mathrm{CH}), 128.3(2 \mathrm{CH}), 127.8(\mathrm{CH}), 127.2(\mathrm{CH}), 42.7\left(\mathrm{CH}_{3}\right) \mathrm{ppm}$; MS (EI), $\mathrm{m} / \mathrm{z}(\%): 178\left(\mathrm{M}^{+}-\mathrm{HSO}_{2} \mathrm{Me}, 100\right)$; HRMS (EI) for $\mathrm{C}_{14} \mathrm{H}_{10}\left(\mathrm{M}^{+}-\mathrm{HSO}_{2} \mathrm{Me}\right)$, calcd: 178.0782, found: 178.0781 .

trans-9,10-Dihydrophenanthrene-9,10-dicarboxylic acid dimethyl ester (trans-3e) ${ }^{9}$

Column chromatography $\left(\mathrm{SiO}_{2} ; 1: 10 \mathrm{AcOEt} /\right.$ hexane) yielded trans-3e as a white solid. M.p. $119^{\circ} \mathrm{C} ;{ }^{1} \mathrm{H}$ $\operatorname{NMR}\left(250 \mathrm{MHz}, \mathrm{CDCl}_{3}\right) \delta=7.75(\mathrm{~d}, J=7.7 \mathrm{~Hz}, 2 \mathrm{H}), 7.44-7.22(\mathrm{~m}, 6 \mathrm{H}), 4.41(\mathrm{~s}, 2 \mathrm{H}), 3.56(\mathrm{~s}, 6 \mathrm{H}) \mathrm{ppm}$; ${ }^{13} \mathrm{C} \mathrm{NMR}\left(62.8 \mathrm{MHz}, \mathrm{CDCl}_{3}\right) \delta=171.9(\mathrm{C}), 133.1(\mathrm{C}), 131.4(\mathrm{C}), 129.9(\mathrm{CH}), 128.4(\mathrm{CH}), 127.9(\mathrm{CH})$, $123.9(\mathrm{CH}), 52.4\left(\mathrm{CH}_{3}\right), 46.9(\mathrm{CH}) \mathrm{ppm}$; MS (EI), m/z (\%): 296 (4), 236 (42), 178 (100); HRMS (EI) for $\mathrm{C}_{18} \mathrm{H}_{16} \mathrm{O}_{4}$, calcd: 296.1048 , found: 296.1042.

\section{Methyl trans-9,10-dihydro-10-methyl-9-phenanthrenecarboxylate (trans-3f) ${ }^{10}$}

Column chromatography $\left(\mathrm{SiO}_{2} ; 1: 50 \mathrm{AcOEt} /\right.$ hexane) yielded trans-3f as a colourless oil. ${ }^{1} \mathrm{H}$ NMR (300 $\left.\mathrm{MHz}, \mathrm{CDCl}_{3}\right) \delta=7.88-7.65(\mathrm{~m}, 2 \mathrm{H}), 7.39-7.23(\mathrm{~m}, 6 \mathrm{H}), 3.67(\mathrm{~d}, J=2.8 \mathrm{~Hz}, 1 \mathrm{H}), 3.52(\mathrm{~s}, 3 \mathrm{H}), 3.50(\mathrm{~m}$, $1 \mathrm{H}), 1.15(\mathrm{~d}, J=7.2 \mathrm{~Hz}, 3 \mathrm{H}) \mathrm{ppm} ;{ }^{13} \mathrm{C} \mathrm{NMR}\left(75 \mathrm{MHz}, \mathrm{CDCl}_{3}\right) \delta=173.2(\mathrm{C}), 139.6(\mathrm{C}), 133.5(\mathrm{C})$, $132.2(\mathrm{C}), 131.7(\mathrm{C}), 130.4(\mathrm{CH}), 128.2(\mathrm{CH}), 128.1(\mathrm{CH}), 127.9(\mathrm{CH}), 127.6(\mathrm{CH}), 127.2(\mathrm{CH}), 123.81$ $(\mathrm{CH}), 123.79(\mathrm{CH}), 52.0\left(\mathrm{CH}_{3}\right), 51.4(\mathrm{CH}), 36.3(\mathrm{CH}), 20.7\left(\mathrm{CH}_{3}\right) \mathrm{ppm} ; \mathrm{MS}(\mathrm{EI}), \mathrm{m} / \mathrm{z}(\%): 252(14), 193$ (100); HRMS (EI) for $\mathrm{C}_{17} \mathrm{H}_{16} \mathrm{O}_{2}$, calcd: 252.1150, found: 252.1150 .

\section{Methyl (E)-3-(1,1'-biphenyl-2-yl)-2-butenoate [(E)-4f]}

Column chromatography $\left(\mathrm{SiO}_{2} ; 1: 50 \mathrm{AcOEt} /\right.$ hexane) yielded $(E)-\mathbf{4 f}$ as a colourless oil. ${ }^{1} \mathrm{H}$ NMR (300 $\left.\mathrm{MHz}_{\mathrm{CDCl}}\right) \delta=7.47-7.14(\mathrm{~m}, 9 \mathrm{H}), 5.96(\mathrm{q}, J=1.3 \mathrm{~Hz}, 1 \mathrm{H}), 3.70(\mathrm{~s}, 3 \mathrm{H}), 2.03(\mathrm{~d}, J=1.3 \mathrm{~Hz}, 3 \mathrm{H}) \mathrm{ppm}$; ${ }^{13} \mathrm{C} \mathrm{NMR}\left(75 \mathrm{MHz}, \mathrm{CDCl}_{3}\right) \delta=166.8(\mathrm{C}), 159.6(\mathrm{C}), 142.8(\mathrm{C}), 141.1(\mathrm{C}), 139.6(\mathrm{C}), 130.4(\mathrm{CH}), 128.8$ $(2 \mathrm{CH}), 128.5(\mathrm{CH}), 128.3(3 \mathrm{CH}), 127.2(\mathrm{CH}), 127.2(\mathrm{CH}), 119.8(\mathrm{CH}), 51.0\left(\mathrm{CH}_{3}\right), 20.6\left(\mathrm{CH}_{3}\right) \mathrm{ppm}$; MS (EI), $m / z$ (\%): 252 (8), 193 (100); HRMS (EI) for $\mathrm{C}_{17} \mathrm{H}_{16} \mathrm{O}_{2}$, calcd: 252.1150, found: 252.1150.

\footnotetext{
${ }_{10}^{9}$ Gawroński, J.; Grycz P.; Kwit, M.; Rychlewska, U. Chem. Eur. J. 2002, 8, 4210.

${ }^{1}$ Lewis, F. D.; DeVoe, R. J. J. Org. Chem. 1982, 47, 888.
} 
The conformation of the product $(E)$ was determined by NOE experiments. Irradiation of the signal at $2.03 \mathrm{ppm}$ (Me trans to the olefinic $\mathrm{H}$ ) showed NOE with aromatic hydrogens but not with the signal at 5.96 ppm (olefinic H).

Methyl 1,2,3,5,6,8,9,10-octahydrodicyclopenta[b,h]phenanthrene-5-carboxylate (6a)

Column chromatography $\left(\mathrm{SiO}_{2} ; 1: 15 \mathrm{AcOEt} /\right.$ hexane) yielded $\mathbf{6 a}$ as a colourless oil. ${ }^{1} \mathrm{H}$ NMR $(250 \mathrm{MHz}$, $\left.\mathrm{CDCl}_{3}\right) \delta=7.64(\mathrm{~s}, 1 \mathrm{H}), 7.60(\mathrm{~s}, 1 \mathrm{H}), 7.10(\mathrm{~s}, 2 \mathrm{H}), 3.82(\mathrm{t}, J=5.8 \mathrm{~Hz}, 1 \mathrm{H}), 3.64(\mathrm{~s}, 3 \mathrm{H}), 3.25(\mathrm{dd}, J=$ 15.1, 6.0 Hz, 1H), 3.12-2.85 (m, 9H), 2.19-2.01 (m, 4H) ppm; ${ }^{13} \mathrm{C} \mathrm{NMR}\left(75 \mathrm{MHz}, \mathrm{CDCl}_{3}\right) \delta=174,3(\mathrm{C})$, 144.4 (C), 143.7 (C), 143.5 (C), 143.4 (C), 133.1 (C), 132.6 (C), 132.5 (C), 131.9 (C), 124.5 (CH), 124.4 $(\mathrm{CH}), 120.1(\mathrm{CH}), 119.7(\mathrm{CH}), 52.2\left(\mathrm{CH}_{3}\right), 45.3(\mathrm{CH}), 33.0\left(2 \mathrm{CH}_{2}\right), 32.9\left(\mathrm{CH}_{2}\right), 32.5\left(\mathrm{CH}_{2}\right), 25.6\left(\mathrm{CH}_{2}\right)$ ppm; MS (EI), m/z (\%): 318 (52), 259 (100); HRMS (EI) for $\mathrm{C}_{22} \mathrm{H}_{22} \mathrm{O}_{2}$, calcd: 318.1620, found: 318.1617; IR (CsI): $1737 \mathrm{~cm}^{-1}$.

Methyl (E)-3-[6-(2,3-dihydro-1H-5-indenyl)-2,3-dihydro-1H-5-indenyl]-2-propenoate [(E)-7a]

Column chromatography $\left(\mathrm{SiO}_{2} ; 1: 15 \mathrm{AcOEt} /\right.$ hexane $)$ yielded $(E)-7 \mathbf{a}$ as a colourless oil. ${ }^{1} \mathrm{H}$ NMR $(300$ $\left.\mathrm{MHz}, \mathrm{CDCl}_{3}\right) \delta=7.76(\mathrm{~d}, J=15.9 \mathrm{~Hz}, 1 \mathrm{H}), 7.57(\mathrm{~s}, 1 \mathrm{H}), 7.30-7.20(\mathrm{~m}, 2 \mathrm{H}), 7.16(\mathrm{~s}, 1 \mathrm{H}), 7.04(\mathrm{~d}, J=7.6$ $\mathrm{Hz}, 1 \mathrm{H}), 6.37(\mathrm{~d}, J=15.9 \mathrm{~Hz}, 1 \mathrm{H}), 3.74(\mathrm{~s}, 3 \mathrm{H}), 2.99-2.92(\mathrm{~m}, 8 \mathrm{H}), 2.19-2.07(\mathrm{~m}, 4 \mathrm{H}) \mathrm{ppm} ;{ }^{13} \mathrm{C}$ NMR $\left(75 \mathrm{MHz}, \mathrm{CDCl}_{3}\right) \delta=167.6(\mathrm{C}), 146.8(\mathrm{C}), 144.8(\mathrm{CH}), 144.2(\mathrm{C}), 143.5(\mathrm{C}), 143.3(\mathrm{C}), 142.0(\mathrm{C}), 138.2$ (C), $130.6(\mathrm{C}), 128.0(\mathrm{CH}), 126.4(\mathrm{CH}), 125.7(\mathrm{CH}), 123.9(\mathrm{CH}), 122.2(\mathrm{CH}), 116.9(\mathrm{CH}), 51.5\left(\mathrm{CH}_{3}\right)$, $32.9\left(\mathrm{CH}_{2}\right), 32.8\left(\mathrm{CH}_{2}\right), 32.6\left(\mathrm{CH}_{2}\right), 32.5\left(\mathrm{CH}_{2}\right), 25.5\left(\mathrm{CH}_{2}\right), 25.4\left(\mathrm{CH}_{2}\right) \mathrm{ppm} ; \mathrm{MS}(\mathrm{EI}) . \mathrm{m} / \mathrm{z}(\%): 318(36)$, 259 (100); HRMS (EI) for $\mathrm{C}_{22} \mathrm{H}_{22} \mathrm{O}_{2}$, calcd: 318.1620 , found: 318.1625 .

Methyl (Z)-3-[6-(2,3-dihydro-1H-5-indenyl)-2,3-dihydro-1H-5-indenyl]-2-propenoate [(Z)-7a] Column chromatography $\left(\mathrm{SiO}_{2} ; 1: 15 \mathrm{AcOEt} /\right.$ hexane) yielded $(Z)-7$ a as a colourless oil. ${ }^{1} \mathrm{H}$ NMR (400 $\left.\mathrm{MHz} \mathrm{CDCl}_{3}\right) \delta=7.49(\mathrm{~s}, 1 \mathrm{H}), 7.25-7.18(\mathrm{~m}, 3 \mathrm{H}), 7.10(\mathrm{~d}, J=7.5 \mathrm{~Hz}, 1 \mathrm{H}), 6.83(\mathrm{~d}, J=12.4 \mathrm{~Hz}, 1 \mathrm{H})$, $5.84(\mathrm{~d}, J=12.3 \mathrm{~Hz}, 1 \mathrm{H}), 3.71(\mathrm{~s}, 3 \mathrm{H}), 2.16-2.07(\mathrm{~m}, 8 \mathrm{H}), 3.05-2.84(\mathrm{~m}, 4 \mathrm{H}) \mathrm{ppm} ;{ }^{13} \mathrm{C} \mathrm{NMR}(100 \mathrm{MHz}$, $\left.\mathrm{CDCl}_{3}\right) \delta=167.0(\mathrm{C}), 145.5(\mathrm{C}), 145.2(\mathrm{CH}), 144.0(\mathrm{C}), 143.2(\mathrm{C}), 142.4(\mathrm{C}), 140.4(\mathrm{C}), 138.9(\mathrm{C}), 131.4$ (C), $128.0(\mathrm{CH}), 125.8(\mathrm{CH}), 125.6(\mathrm{CH}), 125.4(\mathrm{CH}), 123.7(\mathrm{CH}), 118.1(\mathrm{CH}), 51.2\left(\mathrm{CH}_{3}\right), 32.9\left(\mathrm{CH}_{2}\right)$, $32.8\left(\mathrm{CH}_{2}\right), 32.6\left(\mathrm{CH}_{2}\right), 32.5\left(\mathrm{CH}_{2}\right), 25.5\left(\mathrm{CH}_{2}\right), 25.4\left(\mathrm{CH}_{2}\right)$ ppm; MS (EI). m/z (\%): $318(29), 259$ (100); HRMS (EI) for $\mathrm{C}_{22} \mathrm{H}_{22} \mathrm{O}_{2}$, calcd: 318.1620 , found: 318.1627 .

Methyl 5,6-dihydro[1,3]dioxolo[4',5':6,7]phenanthro[2,3-d][1,3]dioxole-5-carboxylate (6b)

Column chromatography $\left(\mathrm{SiO}_{2} ; 1: 40 \mathrm{AcOEt} /\right.$ hexane) yielded $\mathbf{6 b}$ as a colourless oil. ${ }^{1} \mathrm{H}$ NMR $(400 \mathrm{MHz}$, $\left.\mathrm{CDCl}_{3}\right) \delta=7.09(\mathrm{~s}, 1 \mathrm{H}), 7.06(\mathrm{~s}, 1 \mathrm{H}), 6.74(\mathrm{~s}, 1 \mathrm{H}), 6.72(\mathrm{~s}, 1 \mathrm{H}), 6.06-5.87(\mathrm{~m}, 4 \mathrm{H}), 3.70(\mathrm{t}, J=5.4 \mathrm{~Hz}$, $1 \mathrm{H}), 3.61(\mathrm{~s}, 3 \mathrm{H}), 3.18(\mathrm{dd}, J=15.3,5.0 \mathrm{~Hz}, 1 \mathrm{H}), 2.99(\mathrm{dd}, J=15.2,5.8 \mathrm{~Hz}, 1 \mathrm{H}) \mathrm{ppm} ;{ }^{13} \mathrm{C}$ NMR $(100$ $\left.\mathrm{MHz}, \mathrm{CDCl}_{3}\right) \delta=173.3(\mathrm{C}), 147.7$ (C), $147.1(\mathrm{C}), 146.5(\mathrm{C}), 146.4$ (C), 128.5 (C), 127.7 (C), 127.6 (C), $126.8(\mathrm{C}), 109.1(\mathrm{CH}), 108.7(\mathrm{CH}), 104.1(\mathrm{CH}), 104.0(\mathrm{CH}), 101.1\left(\mathrm{CH}_{2}\right), 100.9\left(\mathrm{CH}_{2}\right), 52.1\left(\mathrm{CH}_{3}\right), 44.6$ $(\mathrm{CH}), 31.8\left(\mathrm{CH}_{2}\right)$ ppm; MS (EI), m/z (\%): 326 (87), 267 (100); HRMS (EI) for $\mathrm{C}_{18} \mathrm{H}_{14} \mathrm{O}_{6}$, calcd: 326.0790, found: 326.0791 ; IR (CsI): $1732 \mathrm{~cm}^{-1}$.

\section{Methyl (E)-3-[6-(1,3-benzodioxol-5-yl)-1,3-benzodioxol-5-yl]-2-propenoate [(E)-7b]}

Column chromatography $\left(\mathrm{SiO}_{2} ; 1: 40 \mathrm{AcOEt} /\right.$ hexane) yielded $(E)-7 \mathbf{b}$ as a colourless oil. ${ }^{1} \mathrm{H}$ NMR (400 $\left.\mathrm{MHz}, \mathrm{CDCl}_{3}\right) \delta=7.63(\mathrm{~d}, J=15.9 \mathrm{~Hz}, 1 \mathrm{H}), 7.12(\mathrm{~s}, 1 \mathrm{H}), 6.85(\mathrm{~d}, J=7.9 \mathrm{~Hz}, 1 \mathrm{H}), 6.77(\mathrm{~s}, 1 \mathrm{H}), 6.73(\mathrm{~d}, J$ $=1.7 \mathrm{~Hz}, 1 \mathrm{H}), 6.68(\mathrm{dd}, J=7.9,1.7 \mathrm{~Hz}, 1 \mathrm{H}), 6.24(\mathrm{~d}, J=15.9 \mathrm{~Hz}, 1 \mathrm{H}), 6.03(\mathrm{~s}, 2 \mathrm{H}), 6.02(\mathrm{~s}, 2 \mathrm{H}), 3.73$ (s, 3H) ppm; ${ }^{13} \mathrm{C}$ NMR (75 MHz, $\left.\mathrm{CDCl}_{3}\right) \delta=167.5(\mathrm{C}), 149.1(\mathrm{C}), 147.5(\mathrm{C}), 147.3(\mathrm{C}), 147.1$ (C), 143.5 $(\mathrm{CH}), 138.3(\mathrm{C}), 133.5(\mathrm{C}), 126.4(\mathrm{C}), 123.5(\mathrm{CH}), 116.4(\mathrm{CH}), 110.3(\mathrm{CH}), 110.2(\mathrm{CH}), 108.1(\mathrm{CH})$, $105.6(\mathrm{CH}), 101.6\left(\mathrm{CH}_{2}\right), 101.2\left(\mathrm{CH}_{2}\right), 51.5\left(\mathrm{CH}_{3}\right)$ ppm; MS (EI), m/z (\%): 326 (90), 267 (100); HRMS (EI) for $\mathrm{C}_{18} \mathrm{H}_{14} \mathrm{O}_{6}$, calcd: 326.0790 , found: 326.0783 .

Methyl (Z)-3-[6-(1,3-benzodioxol-5-yl)-1,3-benzodioxol-5-yl]-2-propenoate [(Z)-7b]

Column chromatography $\left(\mathrm{SiO}_{2} ; 1: 40 \mathrm{AcOEt} /\right.$ hexane) yielded $(E)-7 \mathbf{b}$ as a yellowish oil. ${ }^{1} \mathrm{H}$ NMR $(300$ $\left.\mathrm{MHz}, \mathrm{CDCl}_{3}\right) \delta=7.17(\mathrm{~s}, 1 \mathrm{H}), 6.83-6.68(\mathrm{~m}, 5 \mathrm{H}), 6.01(\mathrm{~s}, 2 \mathrm{H}), 6.00(\mathrm{~s}, 2 \mathrm{H}), 5.82(\mathrm{~d}, J=12.3 \mathrm{~Hz}, 1 \mathrm{H})$, $3.73(\mathrm{~s}, 3 \mathrm{H}) \mathrm{ppm} ;{ }^{13} \mathrm{C} \mathrm{NMR}\left(75 \mathrm{MHz}, \mathrm{CDCl}_{3}\right) \delta=166.7$ (C), 148.2 (C), 147.3 (C), 147.0 (C), 146.2 (C), $144.1(\mathrm{CH}), 136.4(\mathrm{C}), 134.2(\mathrm{C}), 126.9(\mathrm{C}), 123.6(\mathrm{CH}), 118.0(\mathrm{CH}), 110.3(\mathrm{CH}), 110.0(\mathrm{CH}), 109.3$ $(\mathrm{CH}), 107.9(\mathrm{CH}), 101.3\left(\mathrm{CH}_{2}\right), 101.1\left(\mathrm{CH}_{2}\right), 51.3\left(\mathrm{CH}_{3}\right) \mathrm{ppm} ; \mathrm{MS}(\mathrm{EI}), \mathrm{m} / \mathrm{z}(\%): 326(92), 267$ (100); HRMS (EI) for $\mathrm{C}_{18} \mathrm{H}_{14} \mathrm{O}_{6}$, calcd: 326.0790, found: 326.0790 . 
9-(2-Cyanoethyl)-9,10-dihydro-9-phenanthrenecarbonitrile (17)

Column chromatography $\left(\mathrm{SiO}_{2}\right.$, AcOEt:hexanes 1:5) yielded a yellowish oil; ${ }^{1} \mathrm{H}$ NMR $\left(400 \mathrm{MHz}, \mathrm{CDCl}_{3}\right)$ $\delta=7.85(\mathrm{~d}, J=7.8 \mathrm{~Hz}, 1 \mathrm{H}), 7.79(\mathrm{~d}, J=7.8 \mathrm{~Hz}, 1 \mathrm{H}), 7.61(\mathrm{ddd}, J=7.6,1.4,0.5 \mathrm{~Hz}, 1 \mathrm{H}), 7.50(\mathrm{dt}, J=$ 7.6, 1.4 Hz, 1H), 7.44-7.39 (m, 2H), $7.34(\mathrm{dt}, J=7.4,1.3 \mathrm{~Hz}, 1 \mathrm{H}), 7.28(\mathrm{~d}, J=7.4 \mathrm{~Hz}, 1 \mathrm{H}), 3.58(\mathrm{~d}, J=$ $15.3 \mathrm{~Hz}, 1 \mathrm{H}$ ), 3.18 (d, $J=15.3 \mathrm{~Hz}, 1 \mathrm{H}$ ), 2.56 (ddd, $J=16.7,10.0,6.7 \mathrm{~Hz}, 1 \mathrm{H}), 2.27$ (ddd, $J=16.6,9.4$, $6.5 \mathrm{~Hz}, 1 \mathrm{H}), 2.02(\mathrm{~m}, 2 \mathrm{H}) \mathrm{ppm} ;{ }^{13} \mathrm{C} \mathrm{NMR}\left(100 \mathrm{MHz}, \mathrm{CDCl}_{3}\right) \delta=132.3(\mathrm{C}), 132.2(\mathrm{C}), 131.6(\mathrm{C}), 130.1$ $(\mathrm{C}), 129.7(\mathrm{CH}), 129.0(\mathrm{CH}), 128.9(\mathrm{CH}), 128.6(\mathrm{CH}), 128.5(\mathrm{CH}), 126.8(\mathrm{CH}), 125.2(\mathrm{CH}), 124.0(\mathrm{CH})$, $121.3(\mathrm{C}), 117.9(\mathrm{C}), 41.6(\mathrm{C}), 38.4\left(\mathrm{CH}_{2}\right), 31.9\left(\mathrm{CH}_{2}\right), 13.8\left(\mathrm{CH}_{2}\right) \mathrm{ppm}$; MS (EI), $\mathrm{m} / \mathrm{z}(\%): 258(26), 204$ (100). 


\section{Experiment with deuterium-labeled methyl acrylate $\left(\boldsymbol{\beta}, \boldsymbol{\beta}-\boldsymbol{d}_{2}-2 \mathrm{a}\right)$}
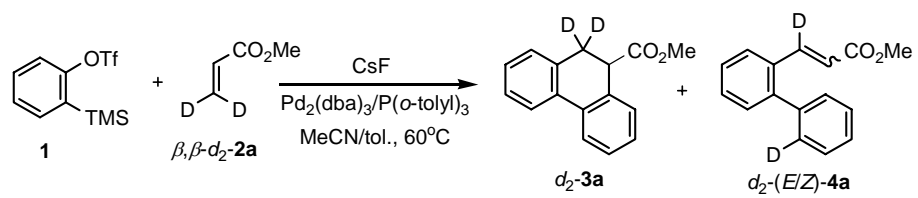

Scheme S2

Isolation of deuterium labeled $d_{2}$-3a was confirmed by comparison of the ${ }^{1} \mathrm{H}$ NMR spectra of $d_{2}$-3a and 3a (Figure S3), showing the lack of two benzylic signals in the spectrum of the deuterated compound.

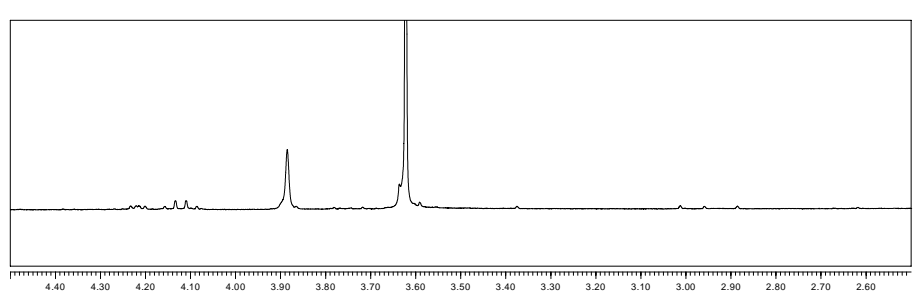<smiles>[2H]C([2H])([2H])C1c2ccccc2-c2ccccc2C1([2H])[2H]</smiles>

ppm (11)
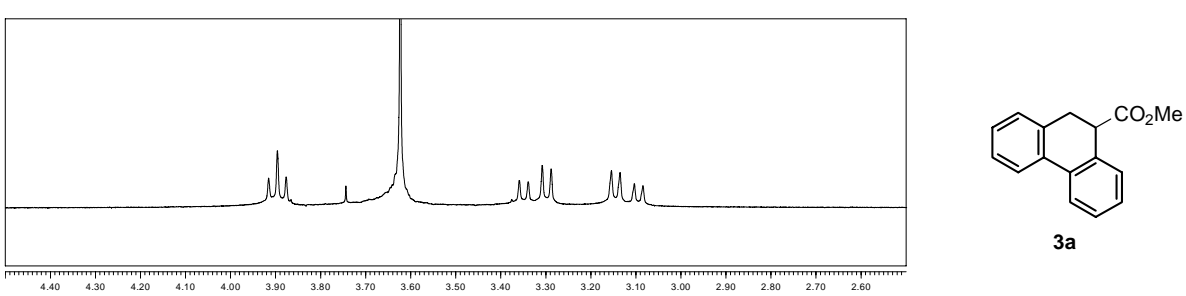

Figure S3

Isolation of $d_{2}-(E / Z)-4 a$ was confirmed by comparison of the ${ }^{13} \mathrm{C}$ NMR (DEPT-135) spectra with those corresponding to (E/Z)-4a (Figures S4 and S5), showing the lack of one aromatic and one olefinic $\mathrm{CH}$ in the spectra of the deuterated derivatives.
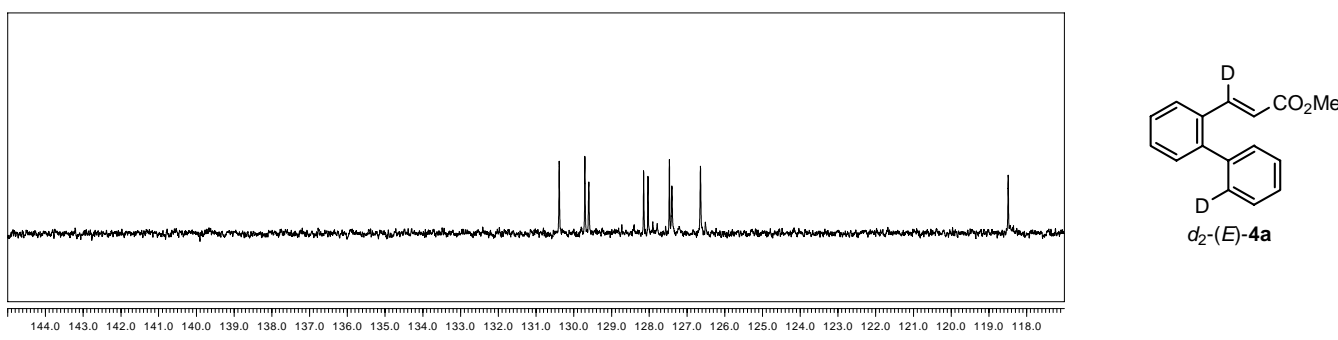

ppm (11)
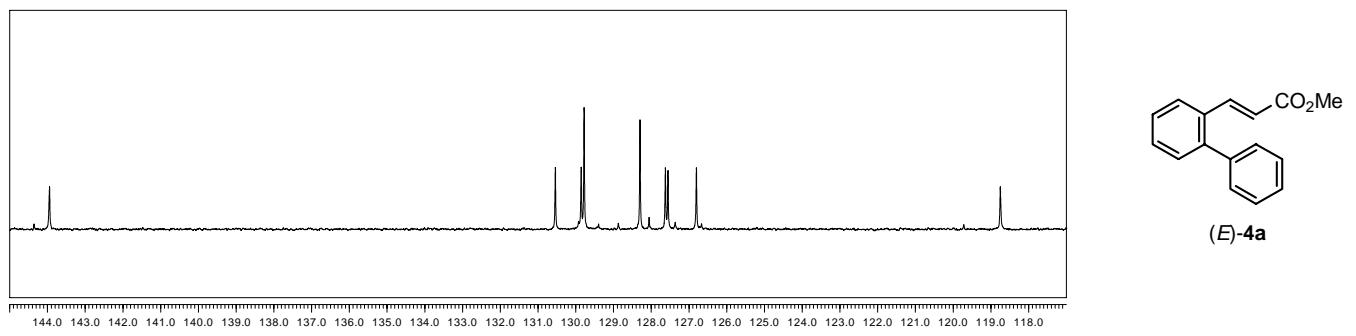

(E)-4a

Figure S4 

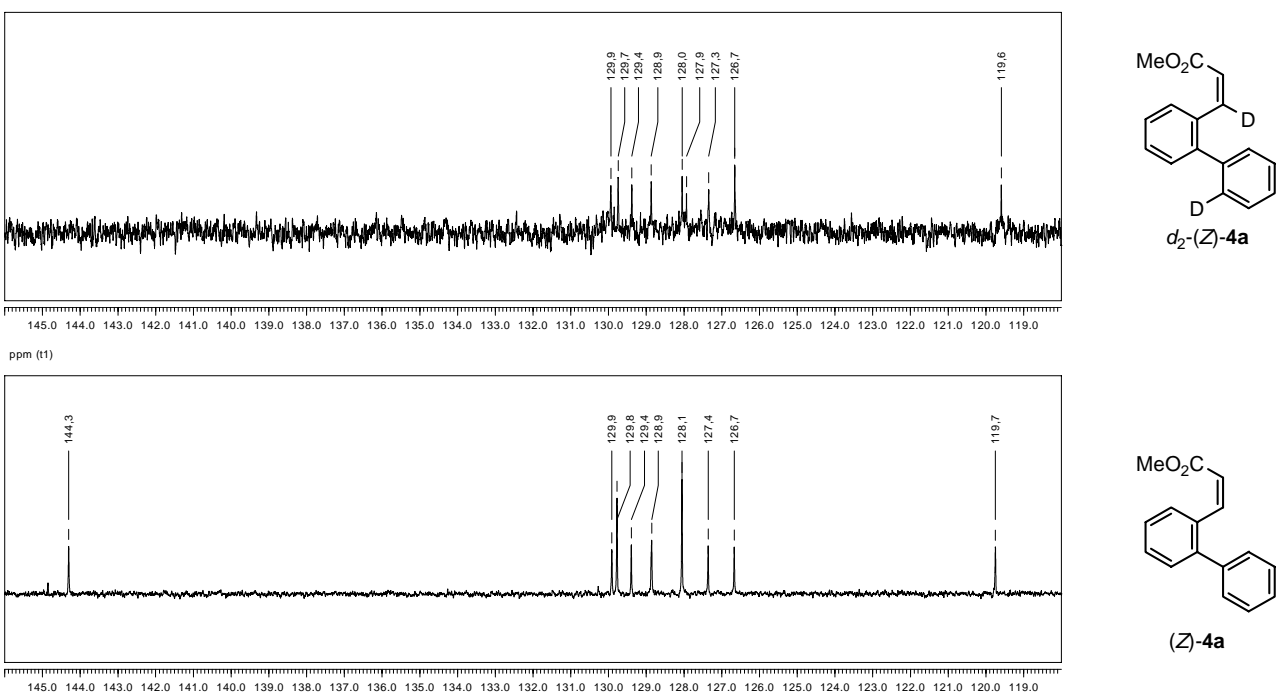

ppm (1t)

Figure S5

The analysis by GC/MS additionally confirmed the isolation of deuterated compounds $d_{2}$-3a and $d_{2}-(E / Z)$ 4a, showing a deuterium incorporation $>98 \%$. 


\section{Experiment using regioisomeric triflates 13a,b}

Figure S6 shows all the possible isomers that would be expected from the cotrimerization of two molecules of 3-methoxybenzyne (14) and one molecule of methyl acrylate (2a). Similarly to the reaction with unsubstituted benzyne, it would be expected four regioisomeric dihydrophenanthrenes (15a-d) and eight $o$-olefinated biaryls $[(E / Z)$-16a-d]. Geminally substituted $o$-olefinated biaryls (i.e. 17) were not isolated from the reaction of benzyne and acrylate. As proposed in this paper, a mechanism via two molecules of benzyne would produce the same isomers from 13a and $\mathbf{1 3 b}$.

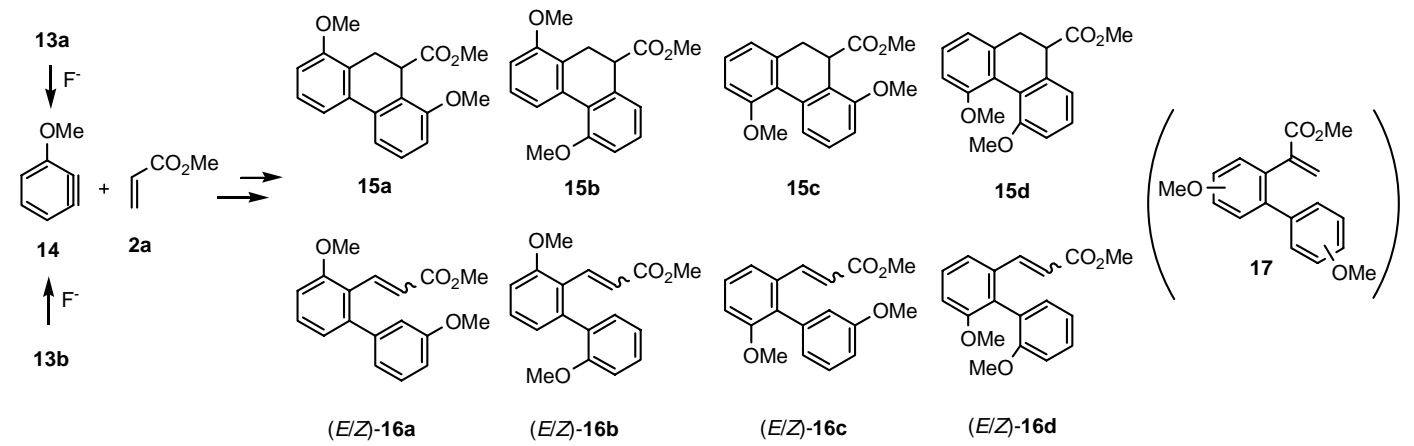

Figure S6

On the contrary, an alternative mechanism initiated by oxidative addition of the aryl triflate to $\operatorname{Pd}(0)$ would produce different isomers from 13a and 13b, as shown in Scheme S3. ${ }^{11}$

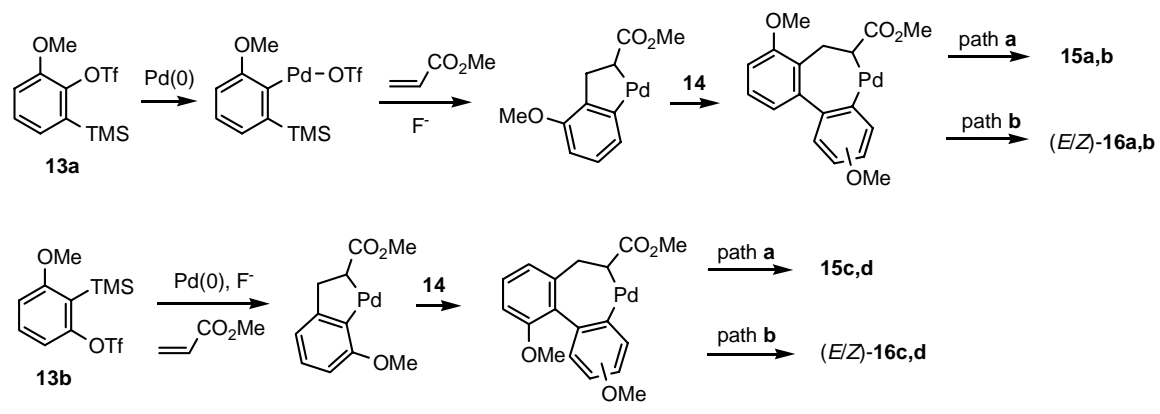

Scheme S3

Analysis by GC/MS of the reactions of acrylate 2a and triflates 13a or 13b, showed identical mixtures of products with $\mathrm{FW}=298$ (15 and 16), with the same isomer ratios for both reactions (Figure S7).
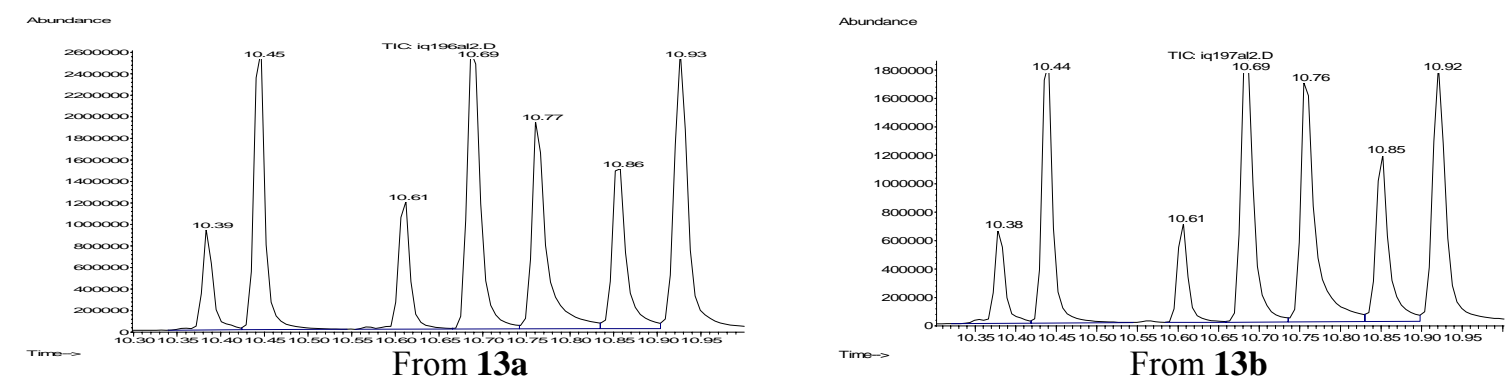

Figure S7

\footnotetext{
11 This alternative has been proposed for some Pd-catalyzed cyclizations of $o$-(trimethylsilyl)phenyl triflates with alkynes. See: Yoshikawa, E.; Radhakrishnan, K. V.; Yamamoto, Y. J. Am. Chem. Soc. 2000, 122, 7280.
} 


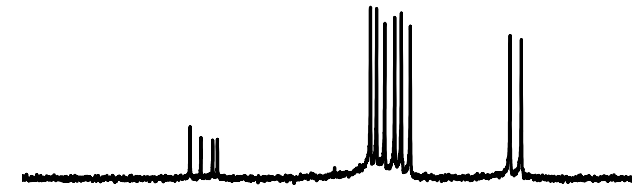

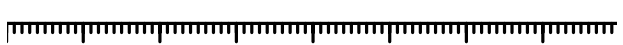

137.5135 .0132 .5130 .0127 .5125 .0122 .5

ppm (t1)

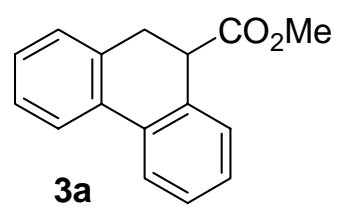

$3 a$
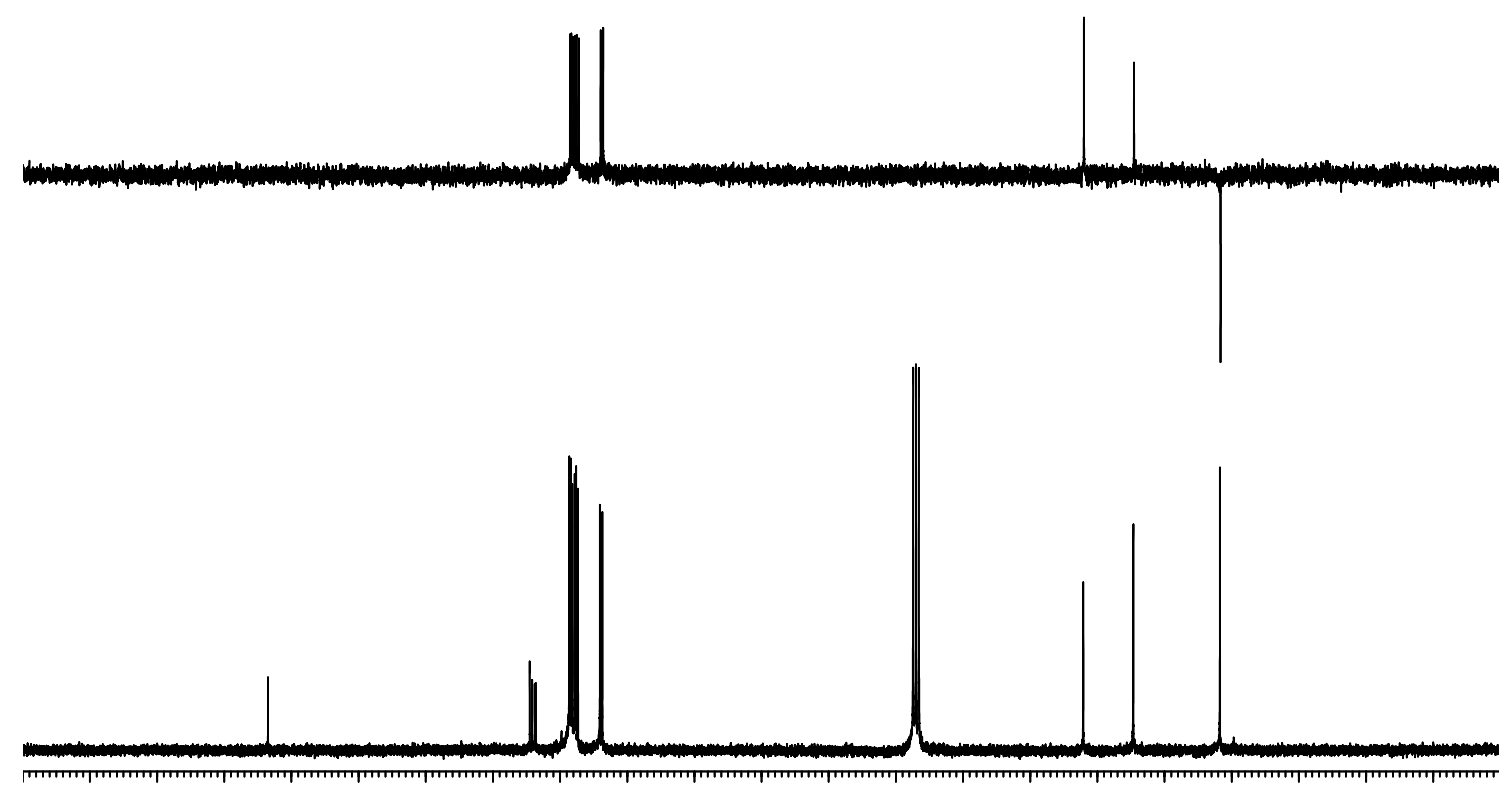
ppm (t1)

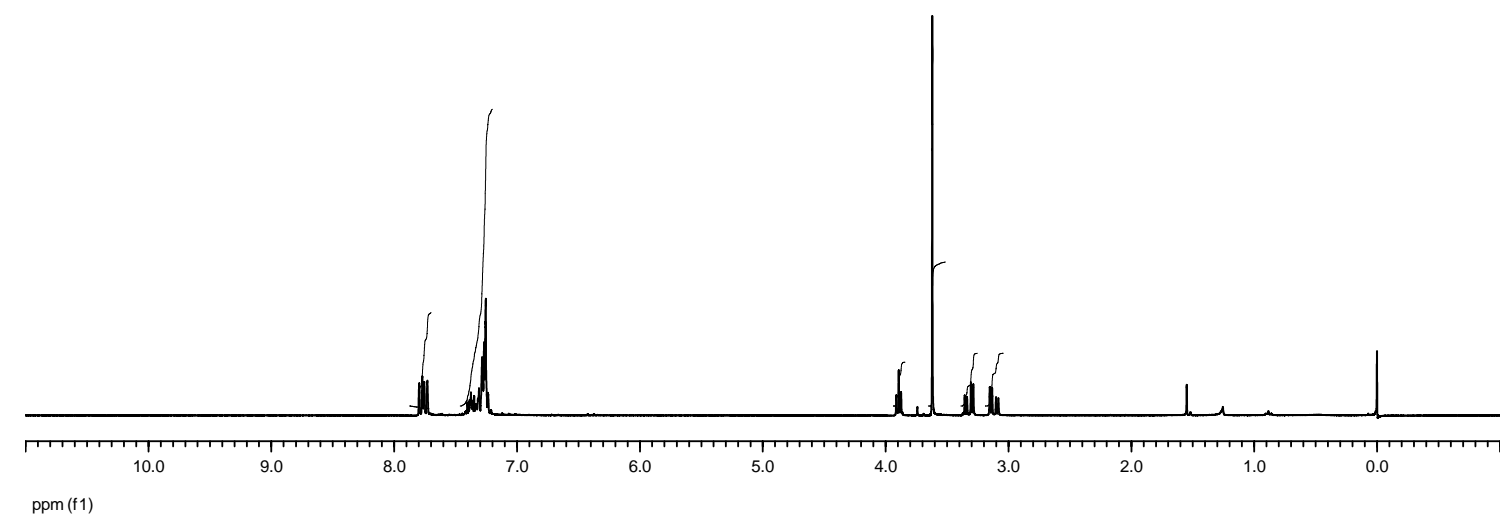




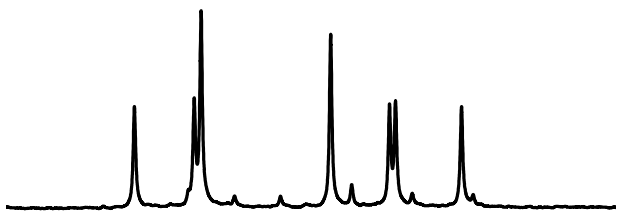

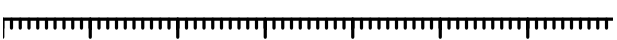

$\begin{array}{llllll}131.0 & 130.0 & 129.0 & 128.0 & 127.0 & 126.0\end{array}$

ppm (t1)
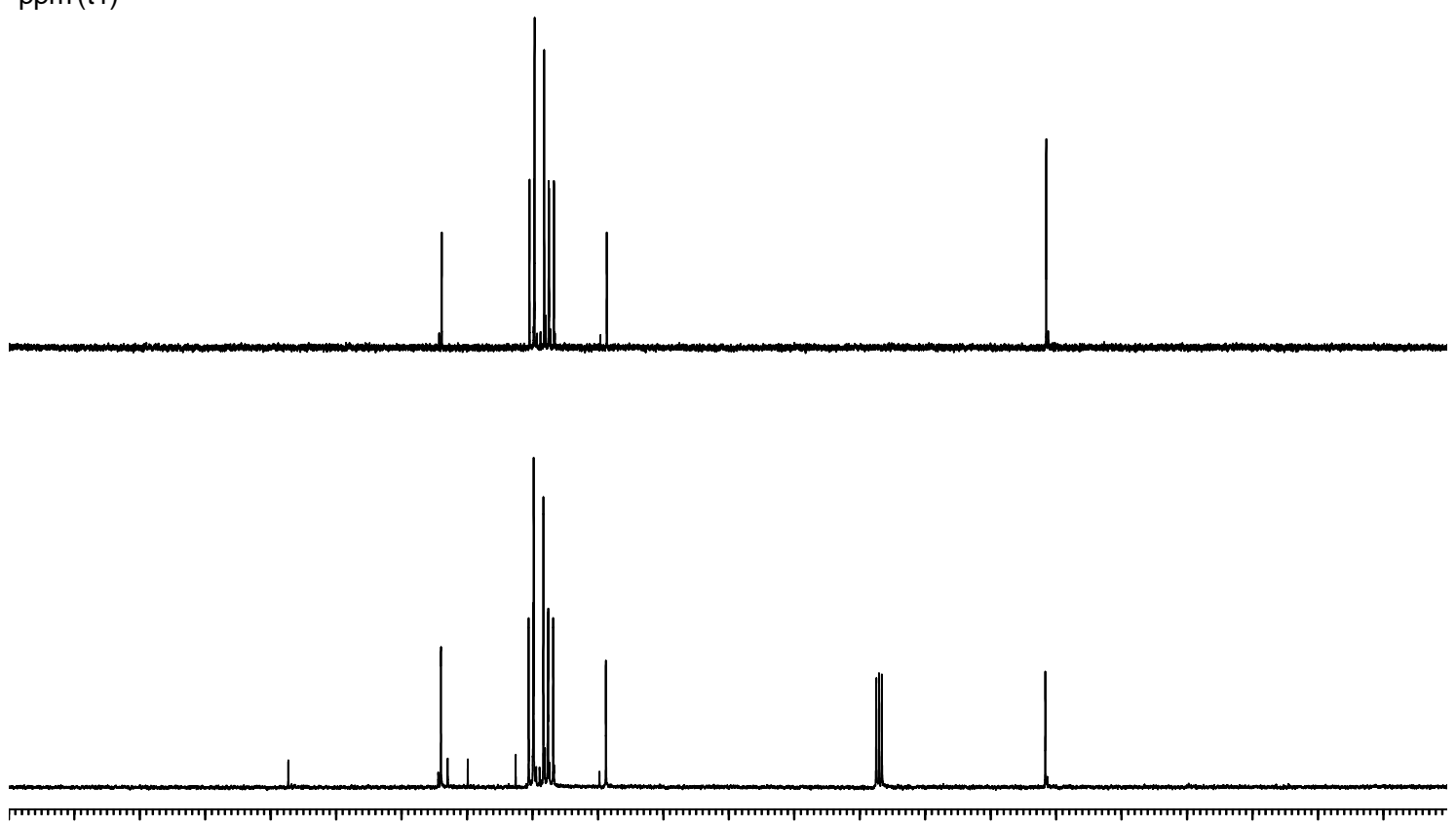
200

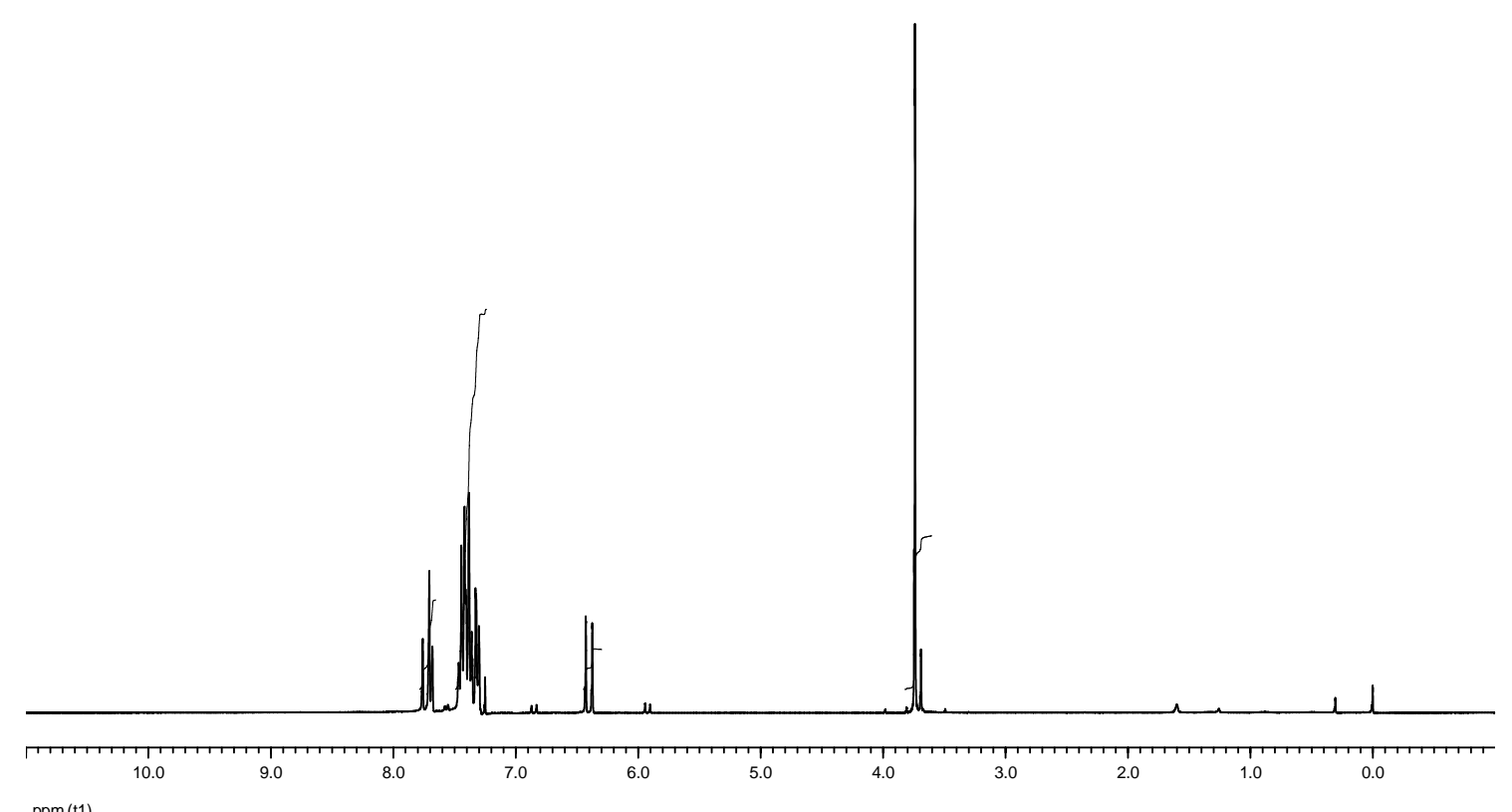

$\operatorname{ppm}(\mathrm{t} 1)$ 


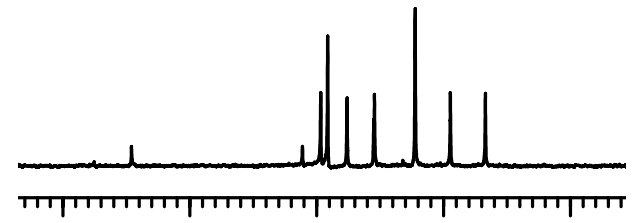

$\begin{array}{lllll}135.0 & 132.5 & 130.0 & 127.5 & 125.0\end{array}$<smiles>COC(=O)/C=C/c1ccccc1-c1ccccc1</smiles>

(Z) $-4 a$

$(20: 80 E / Z)$

ppm (t1)
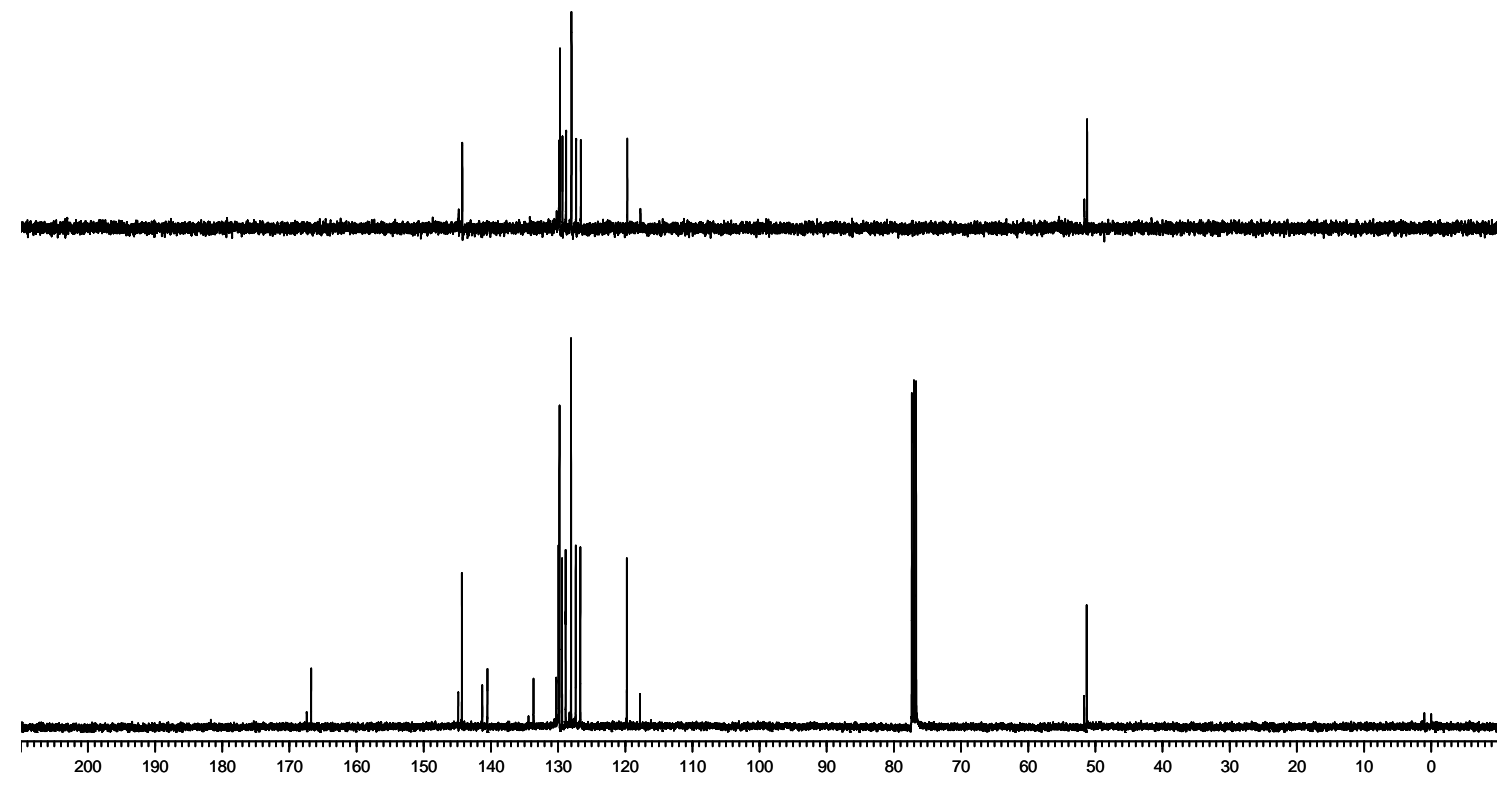
ppm (11)

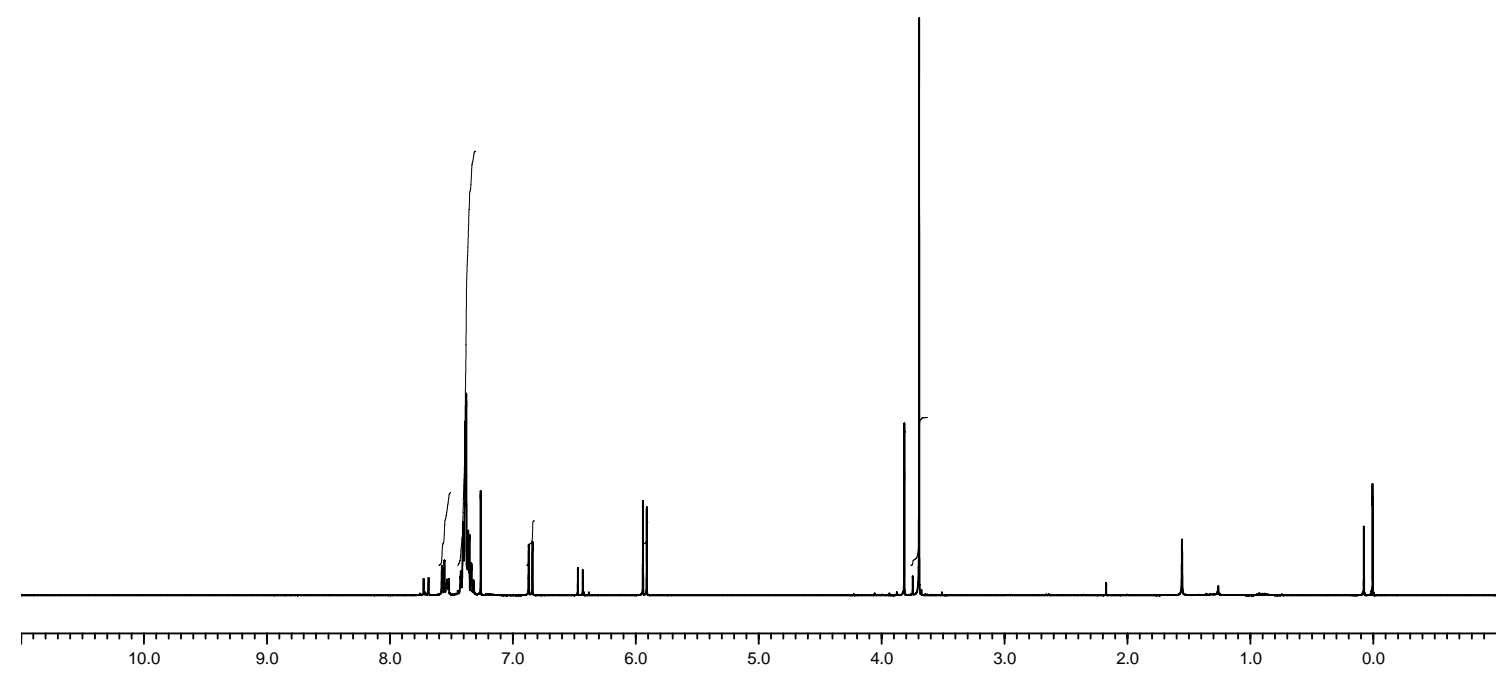
ppm (t1) 

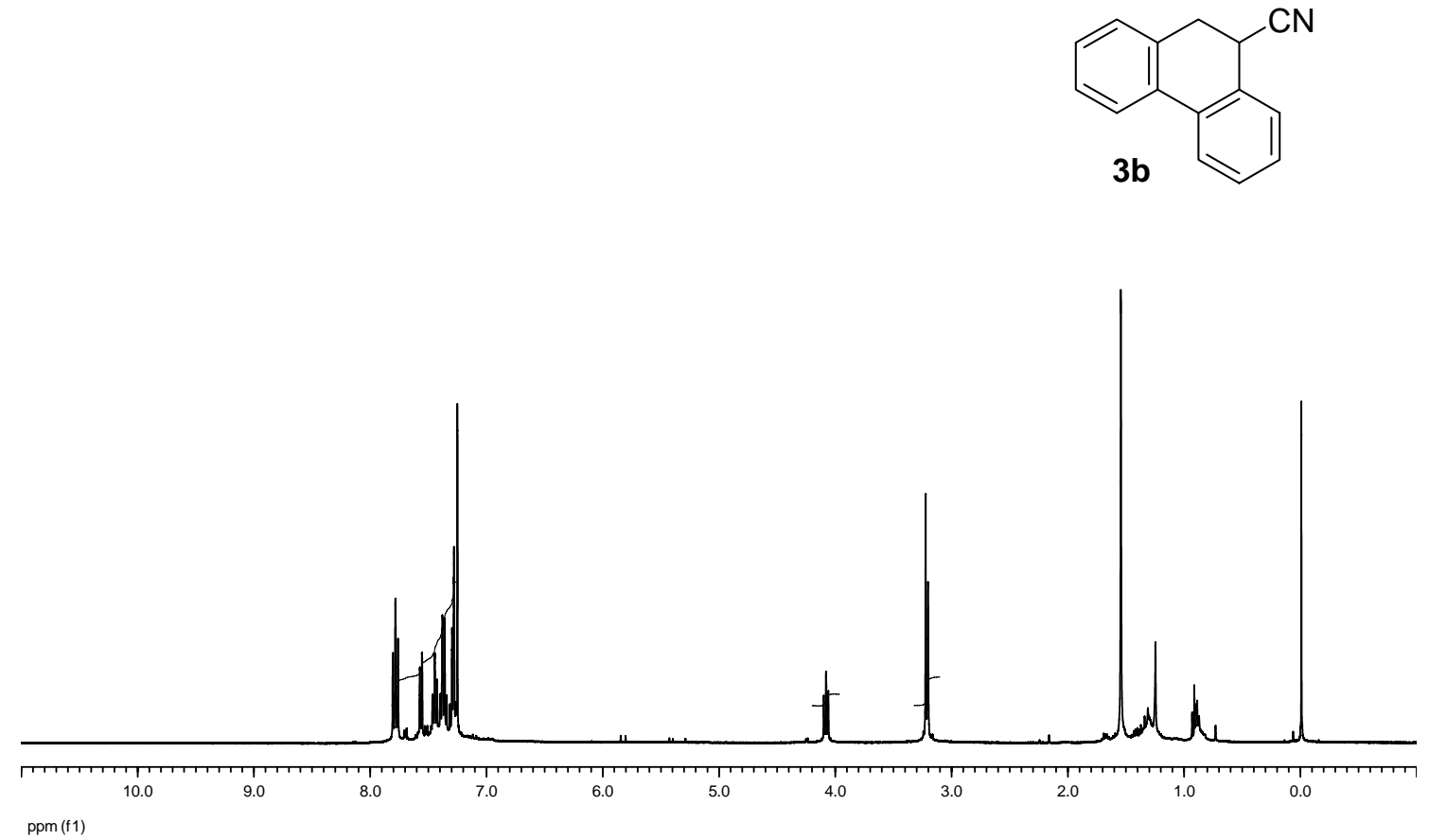


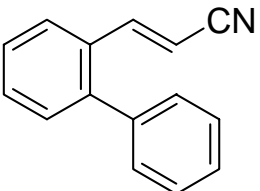

(E)-4b

(96:4 E/Z)
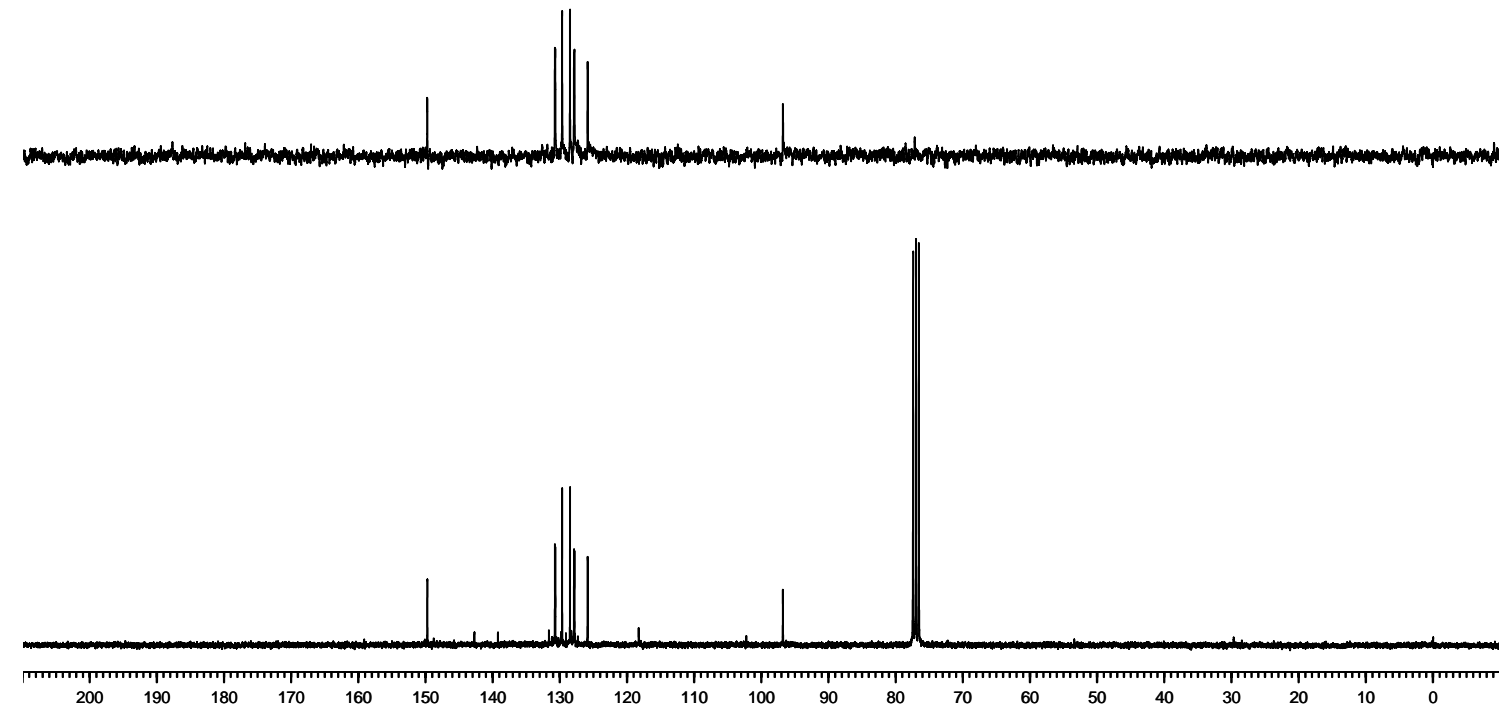

ppm (f1)

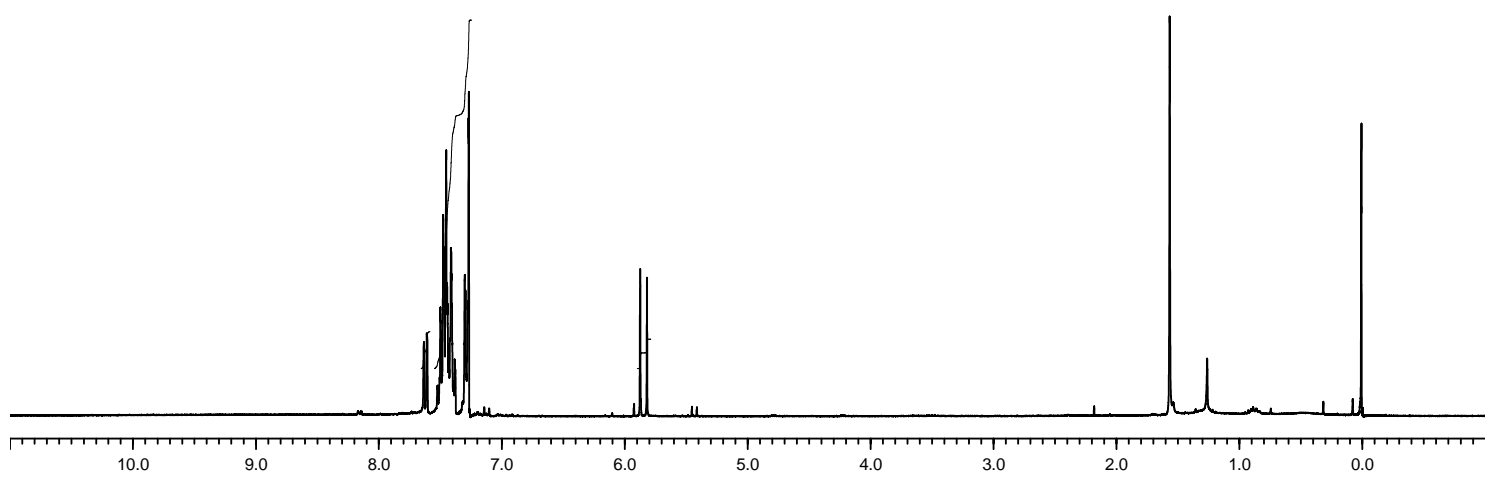

ppm (t1) 
SUPPORTING INFORMATION
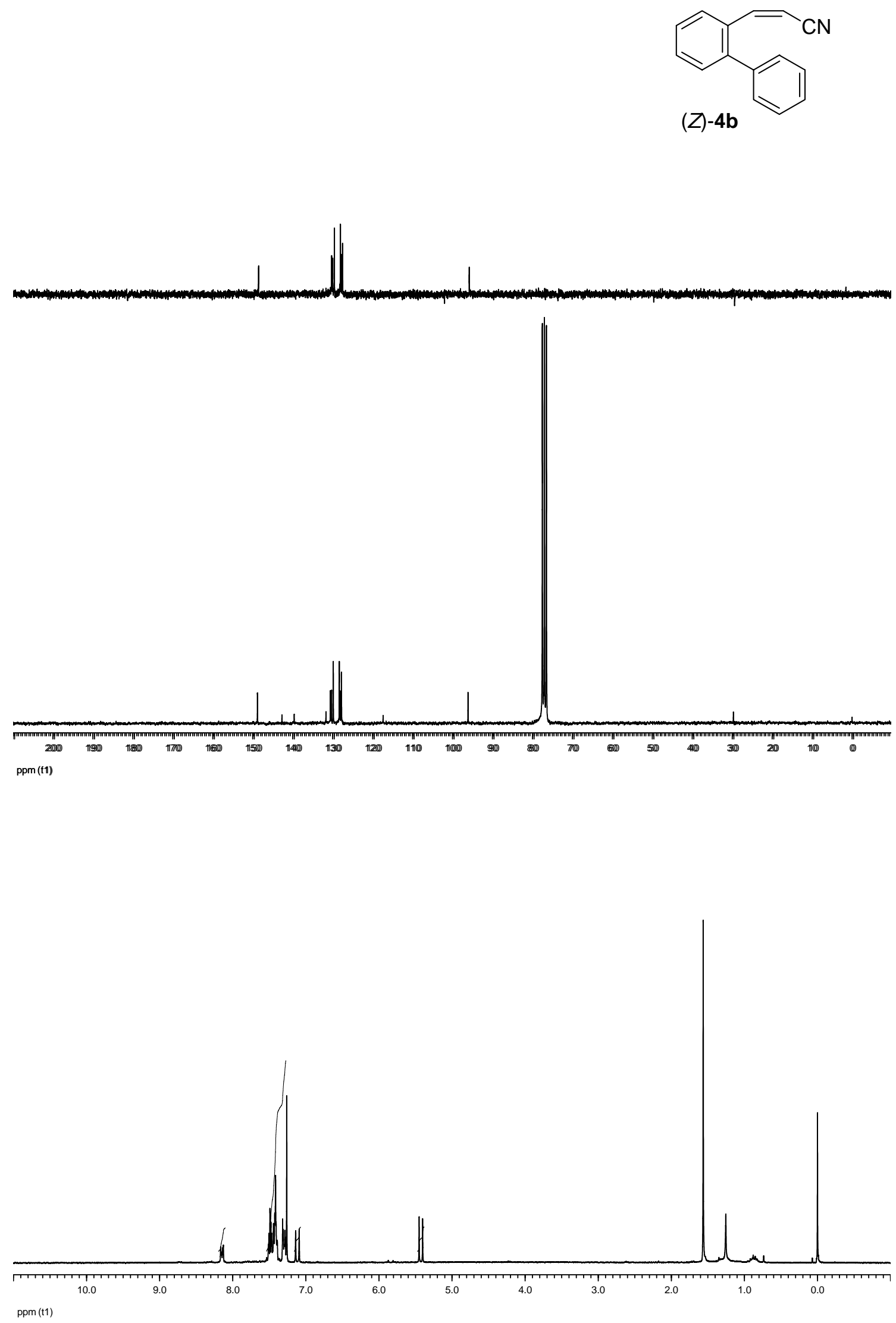

S16 

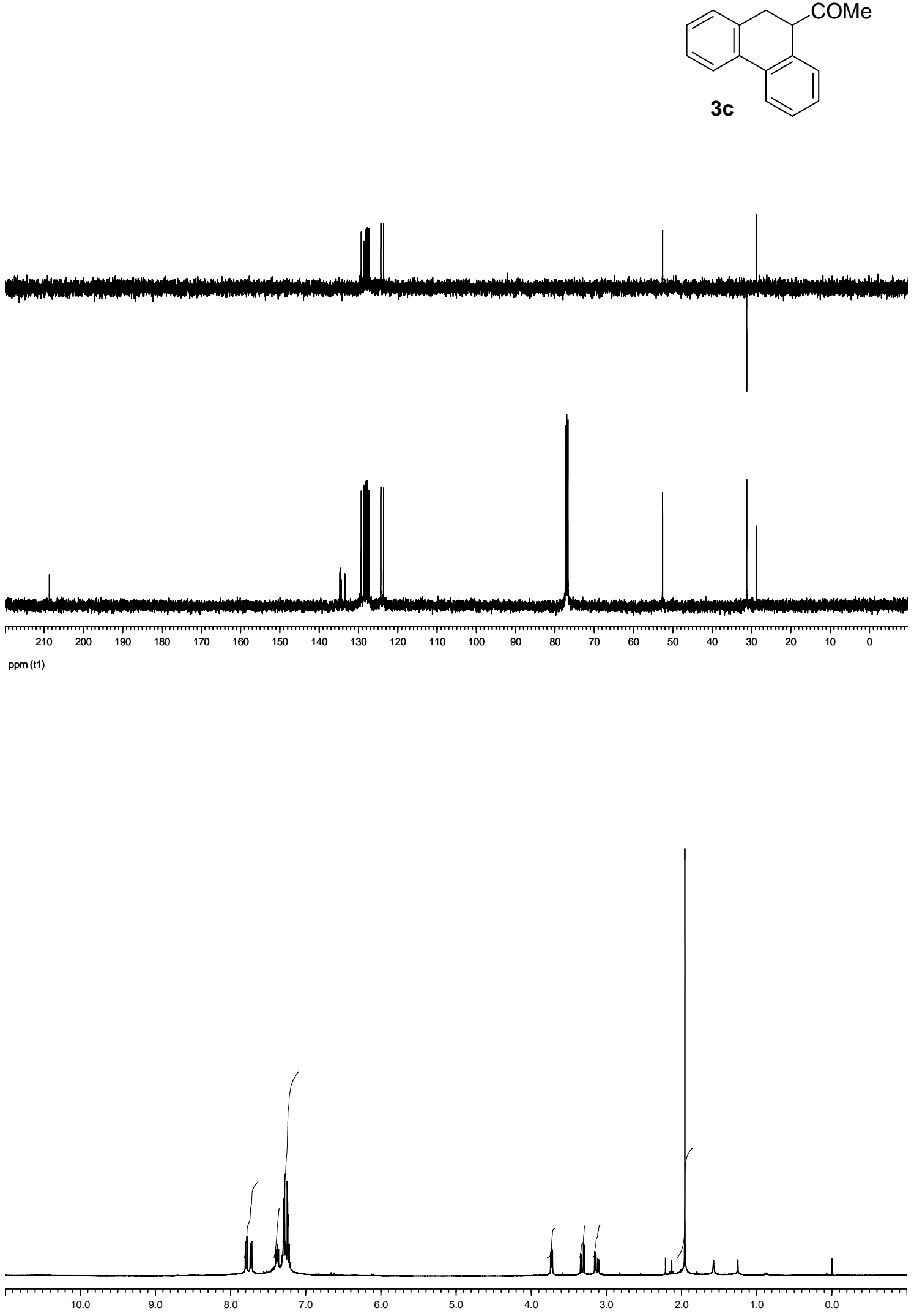

ppm (t1) 
SUPPORTING INFORMATION

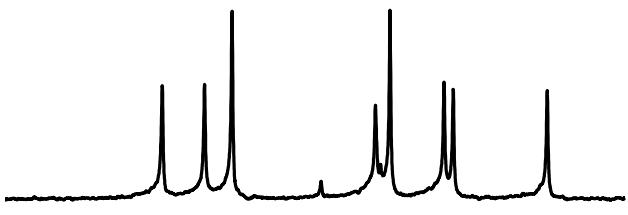

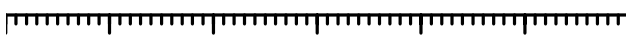
$\begin{array}{lllll}131.0 & 130.0 & 129.0 & 128.0 & 127.0\end{array}$

ppm (t1)

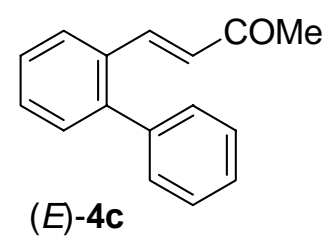

(E)-4c
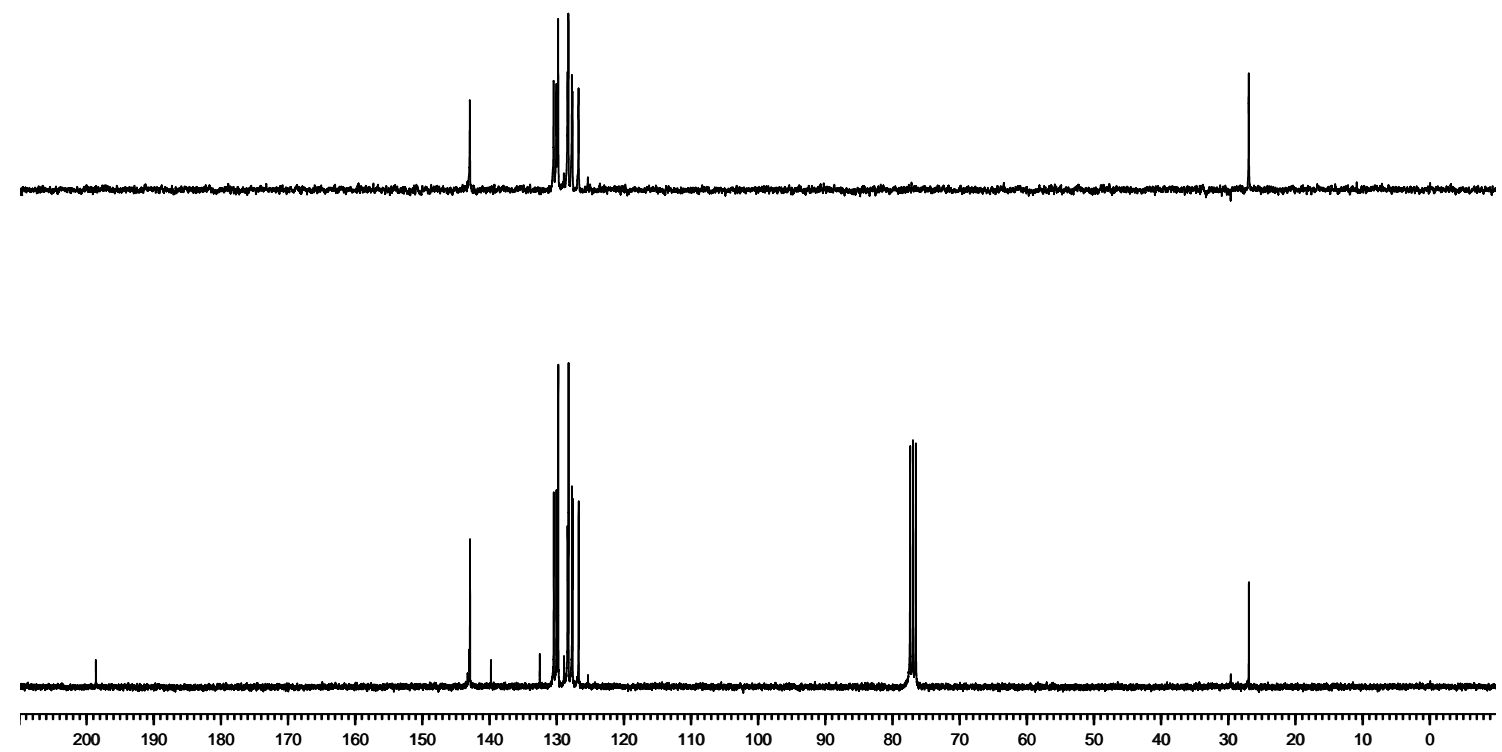

ppm (f1)

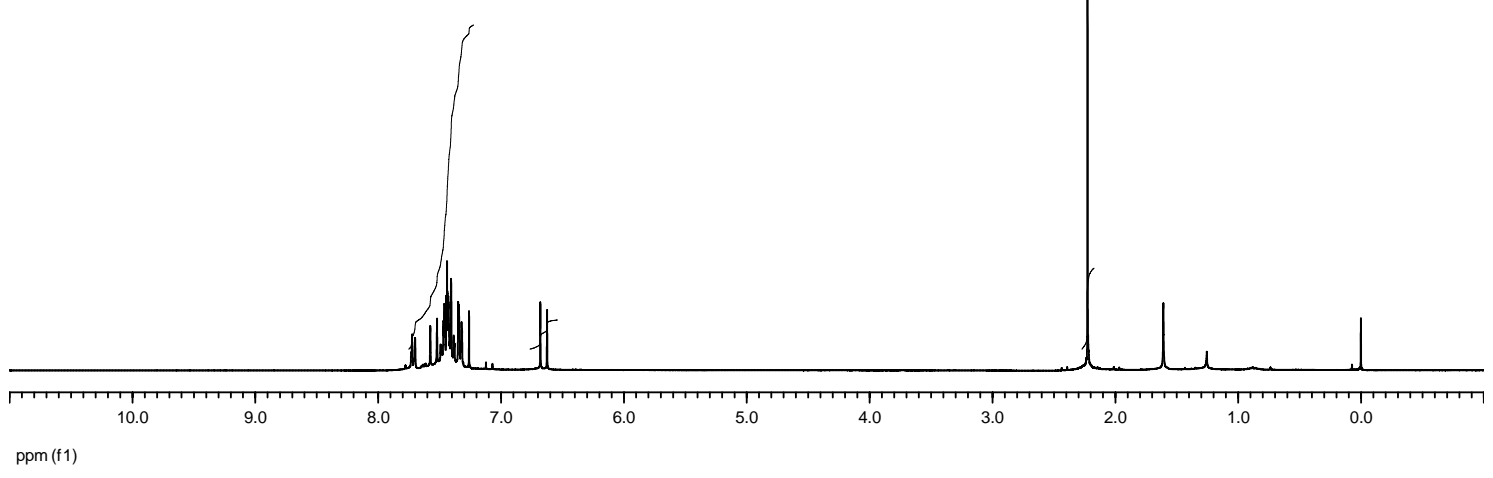

S18 

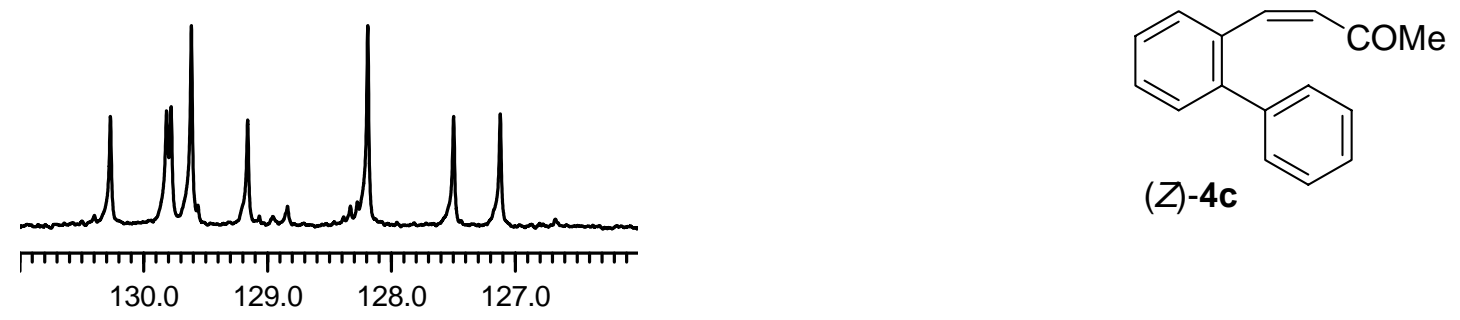

ppm (t1)
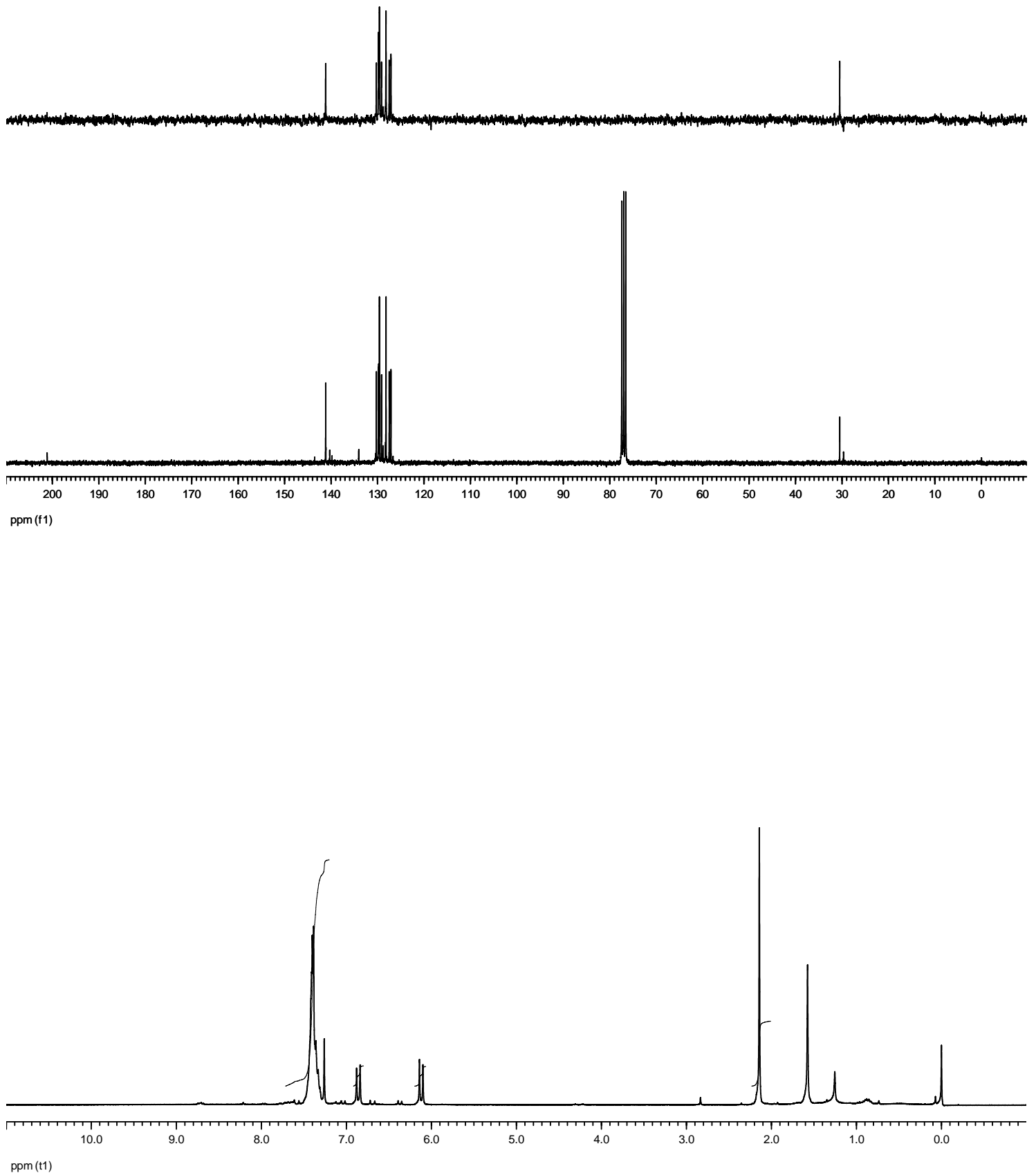

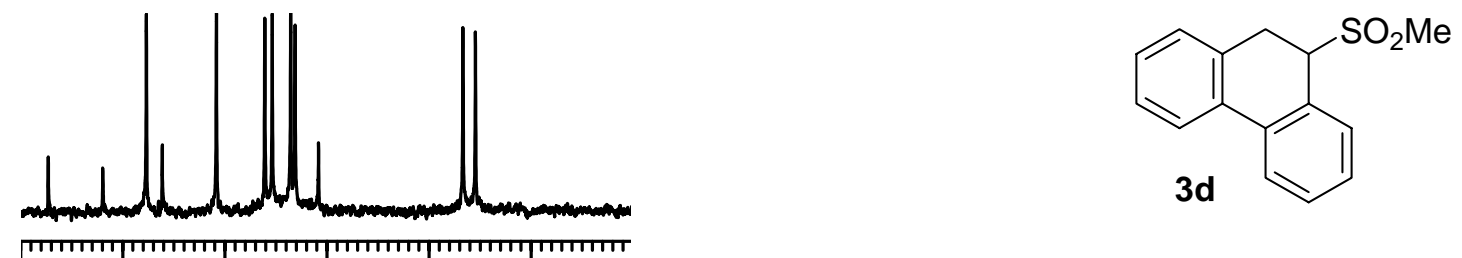

$\begin{array}{lllll}132.5 & 130.0 & 127.5 & 125.0 & 122.5\end{array}$

ppm (t1)
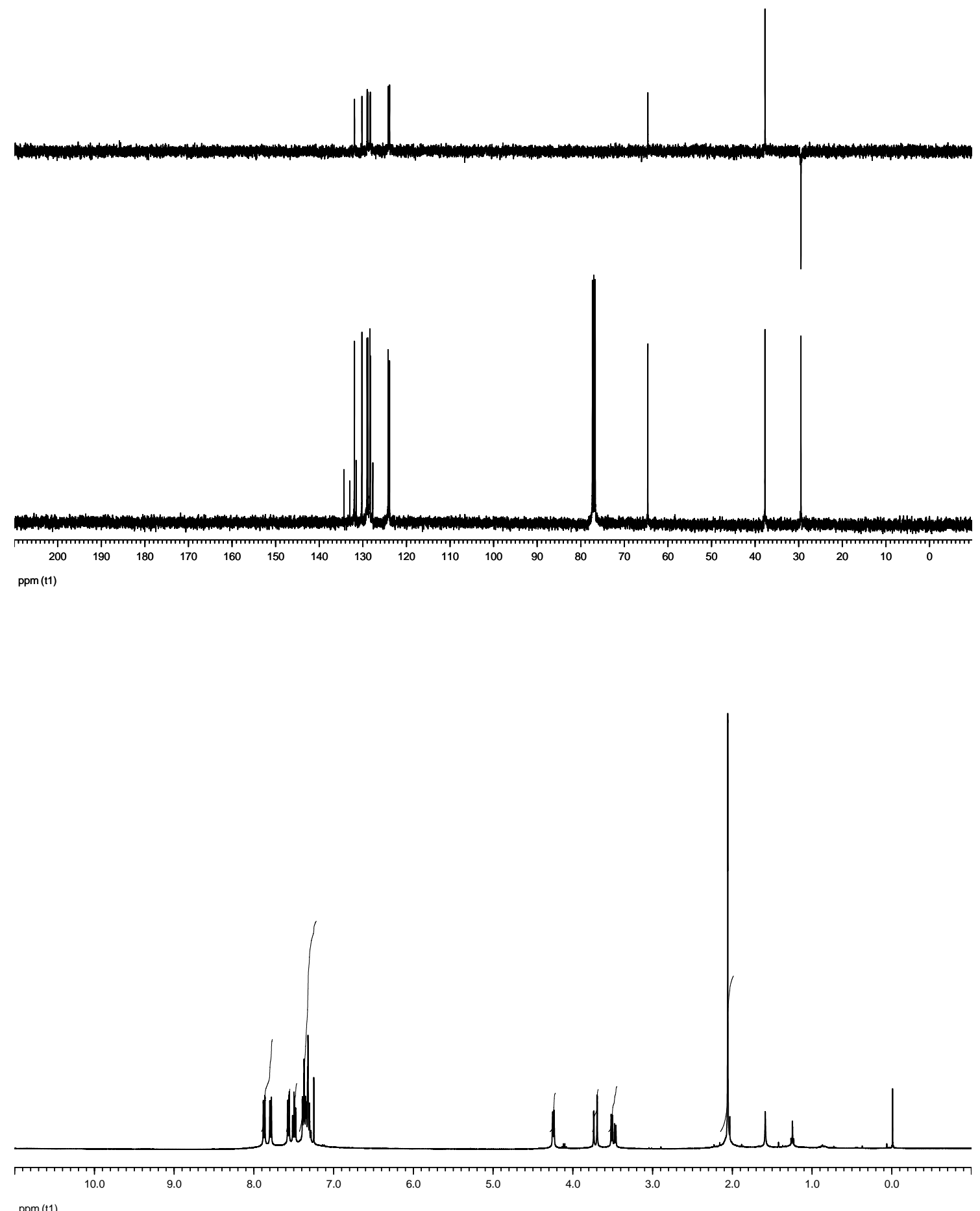
\rfloor$\left._{d}\right\rfloor \|$

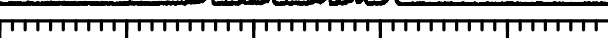

$\begin{array}{llll}132.5 & 130.0 & 127.5 & 125.0\end{array}$

ppm (t1)

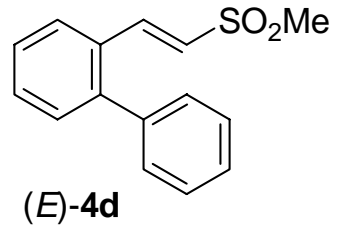

$(E)-\mathbf{4 d}$
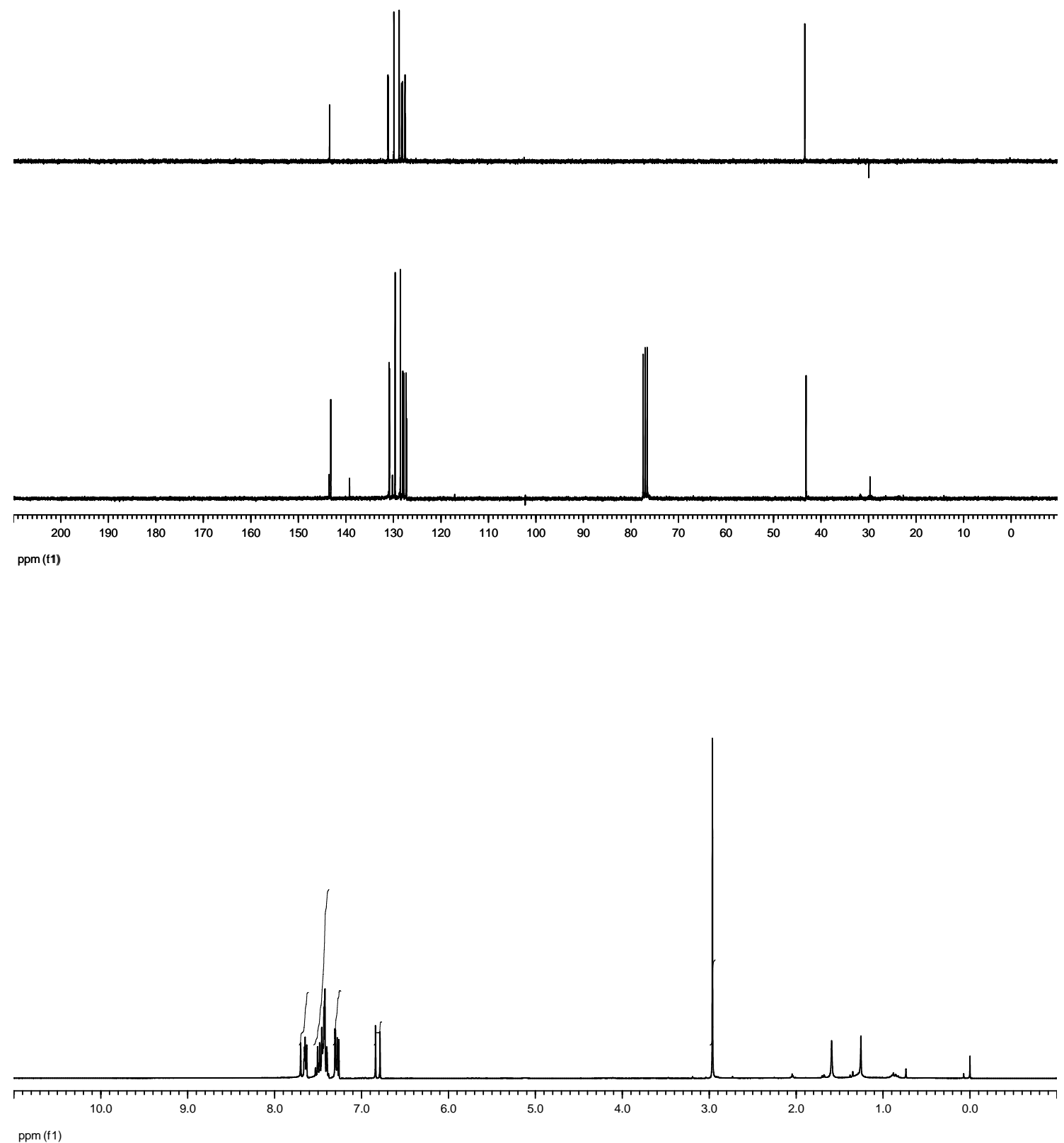


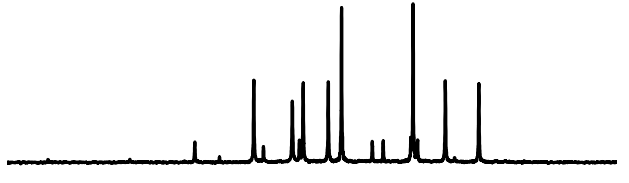
$132.5 \quad 130.0 \quad 127.5$

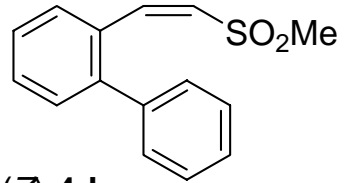

(Z)-4d

[93:7 (Z)-4d/3d]

$\mathrm{ppm}(\mathrm{t} 1)$
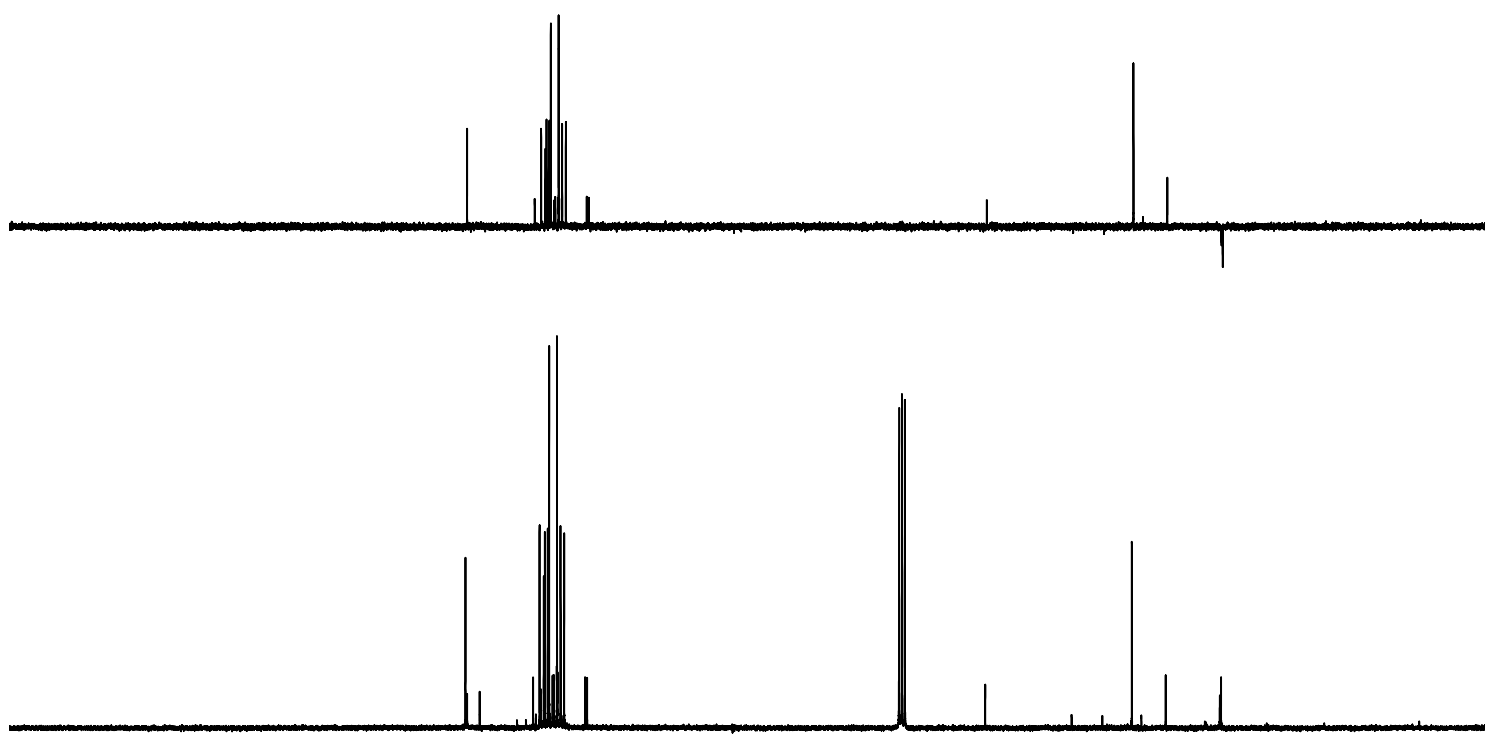

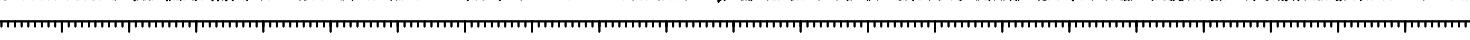
ppm (11)

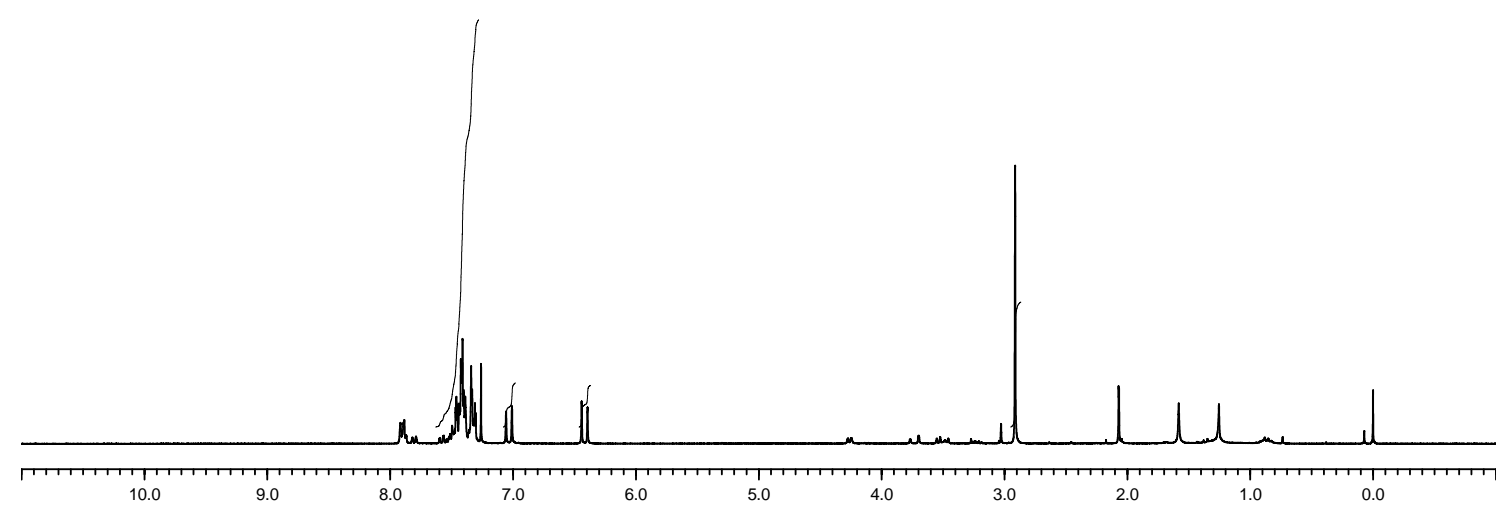

$\operatorname{ppm}($ (f1) 

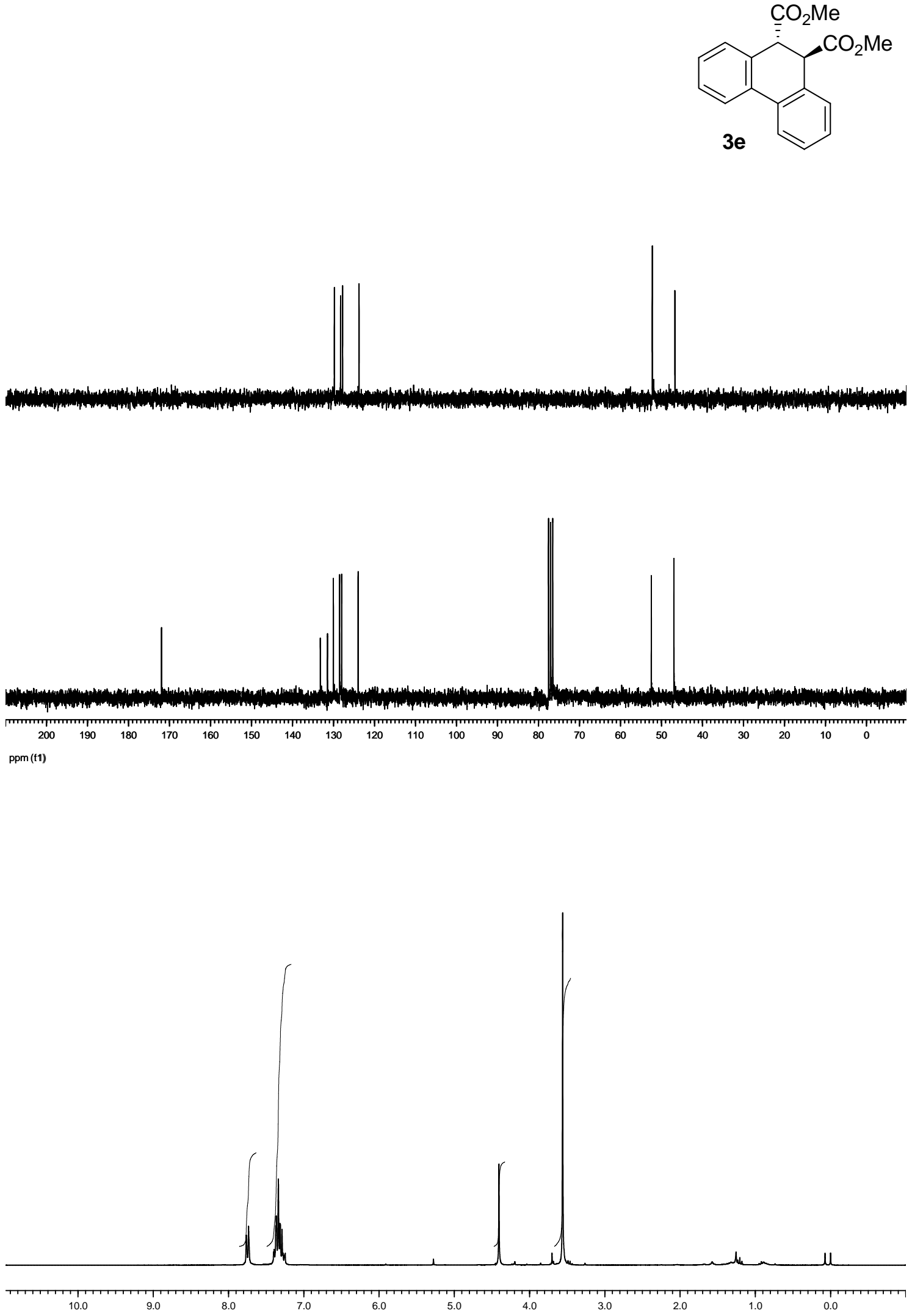

$\operatorname{ppm}(\mathrm{t} 1)$ 


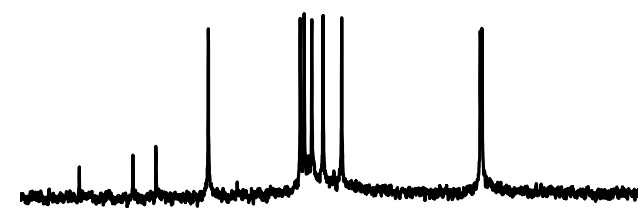

пाтाтाтाтाтाтाтाтाтाтाтाтाтाртाтाтाтाтाтाтा $\begin{array}{lllll}132.5 & 130.0 & 127.5 & 125.0 & 122.5\end{array}$

ppm (t1)

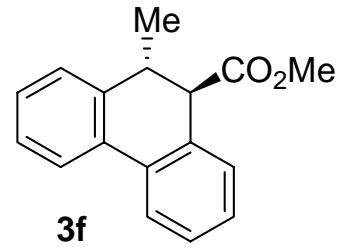

$3 f$
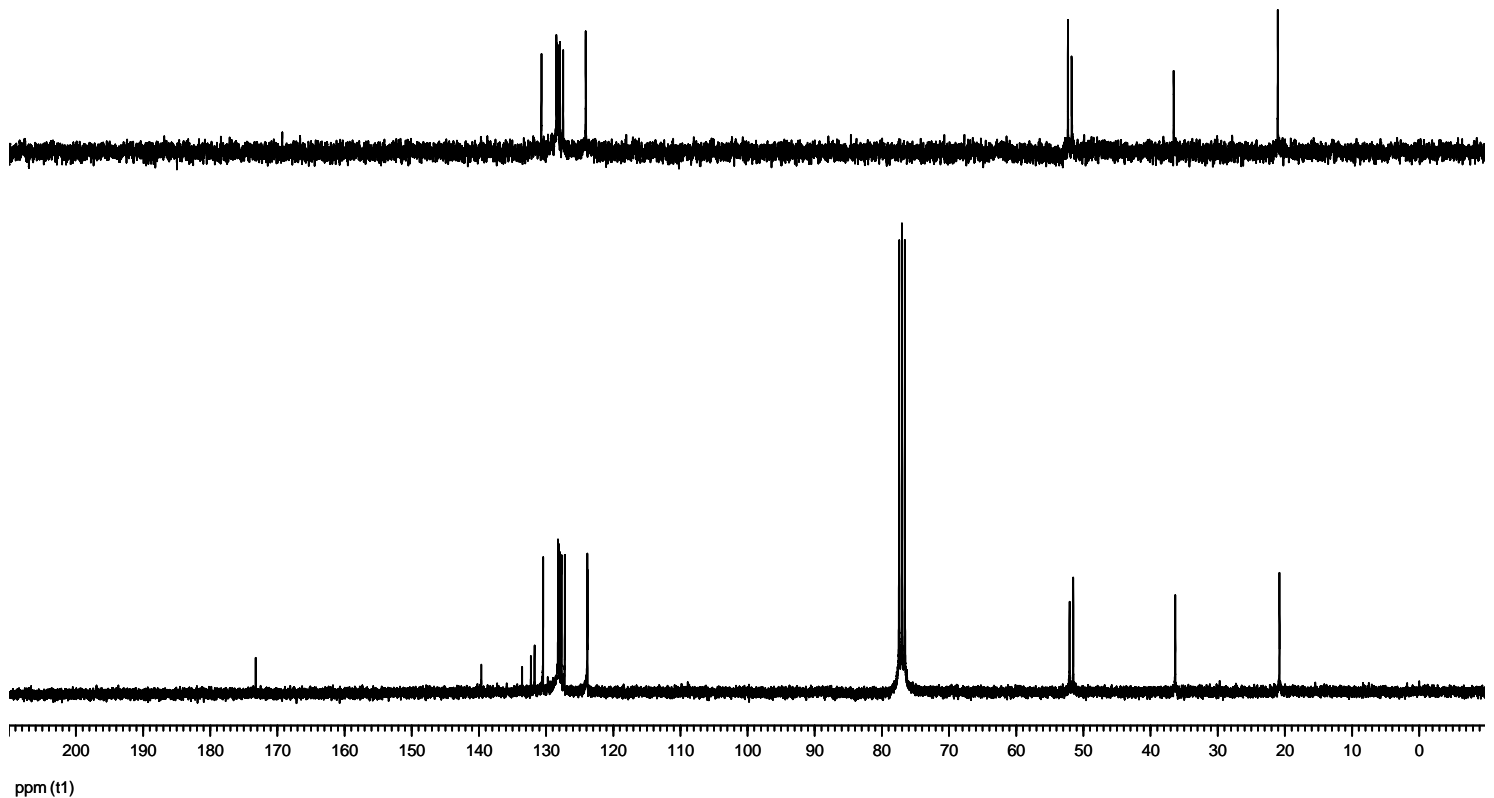

ppm (11)

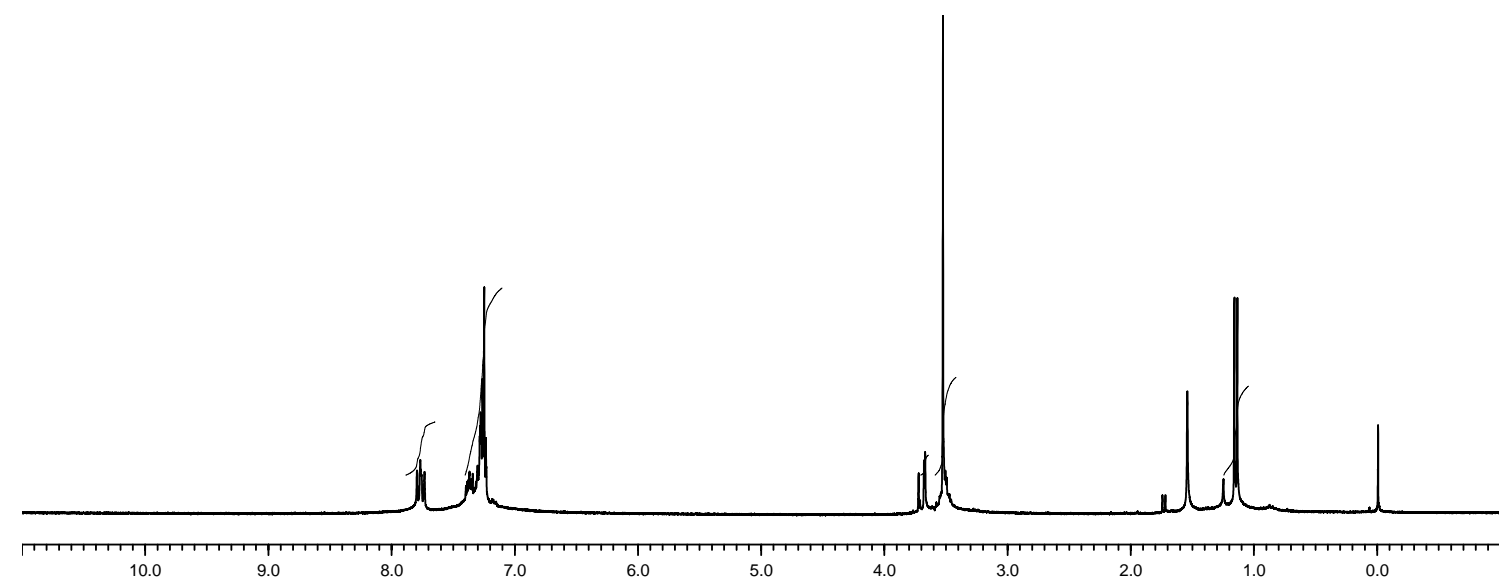

ppm (t1) 
SUPPORTING INFORMATION
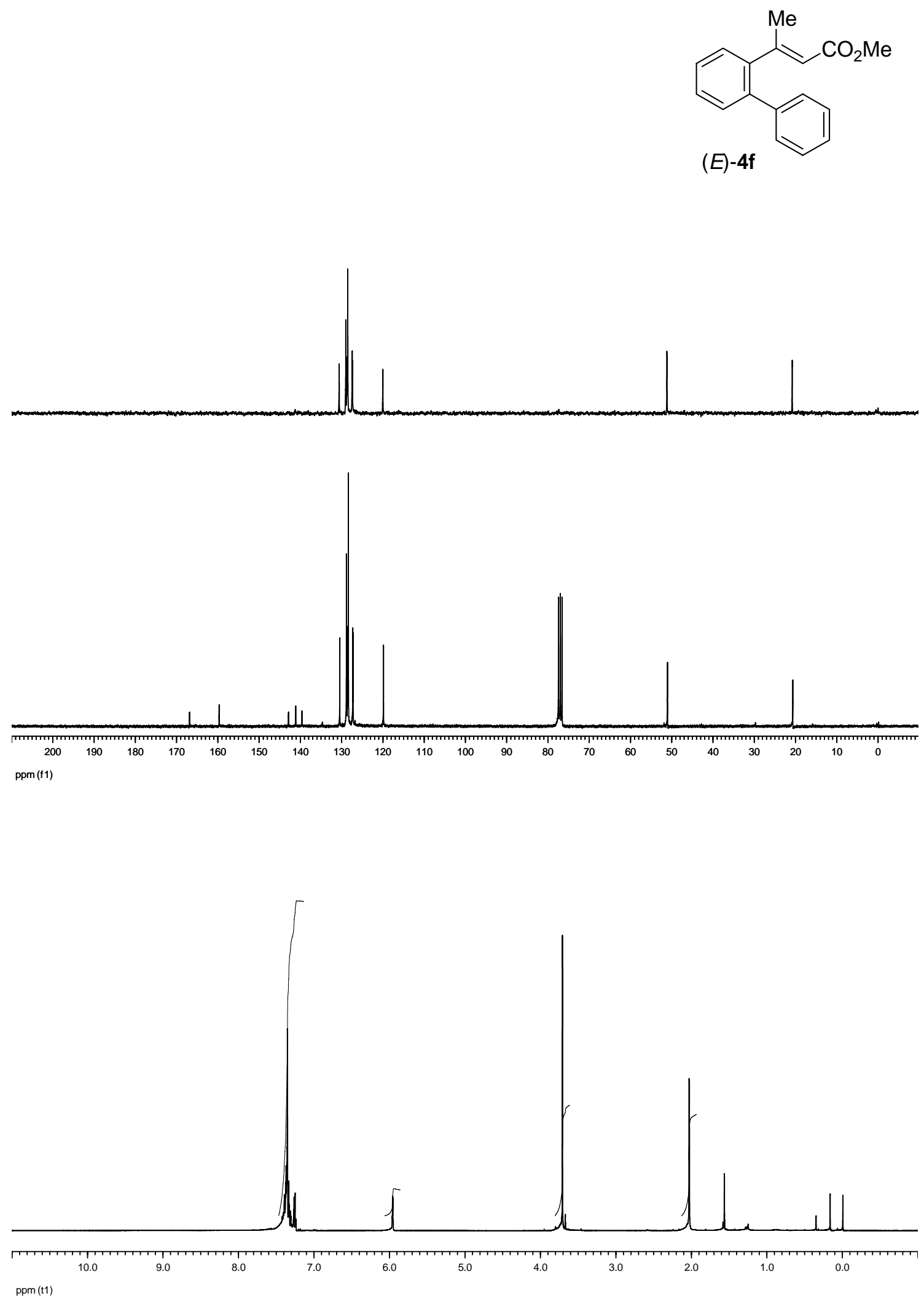

S25 

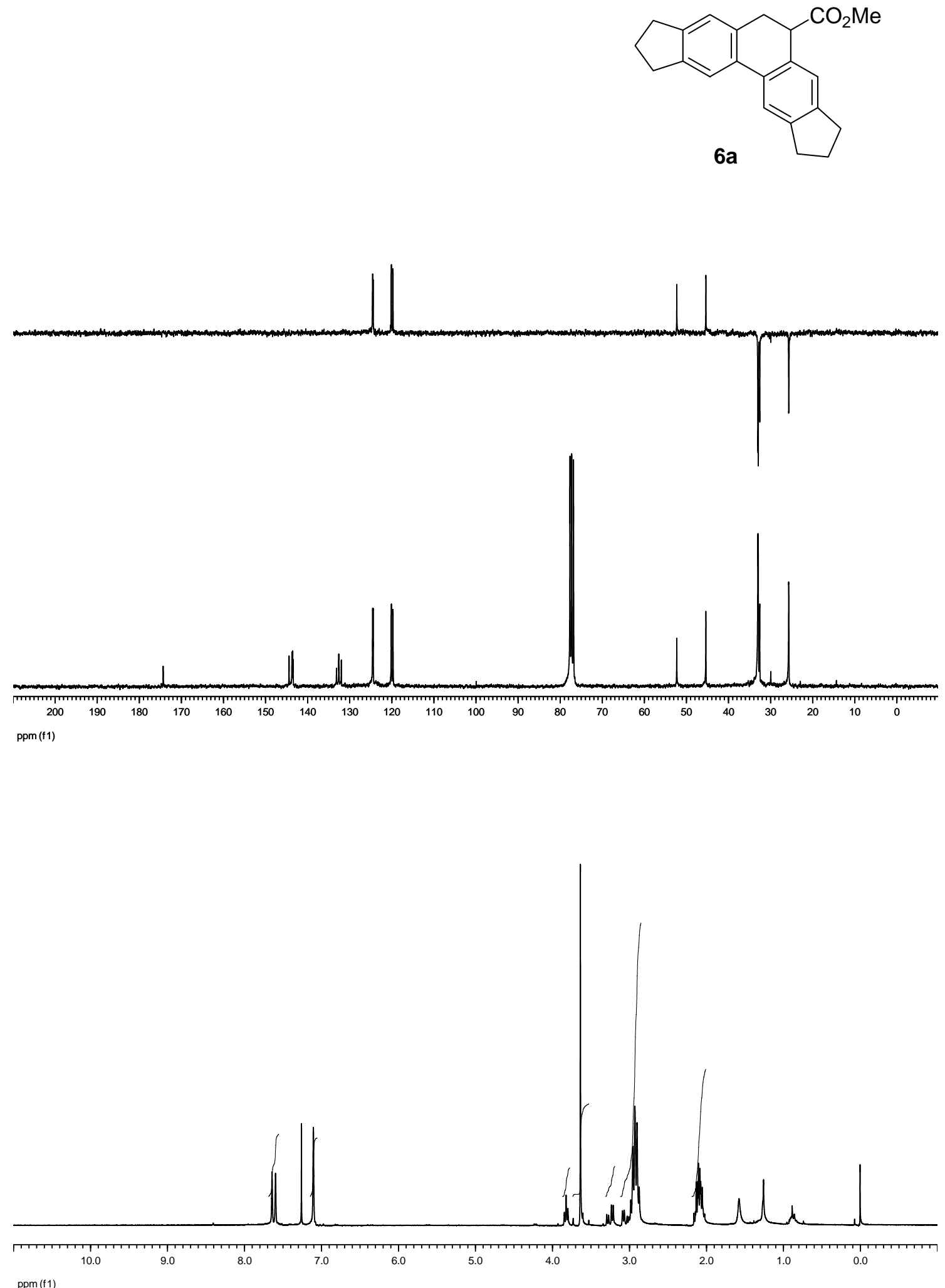


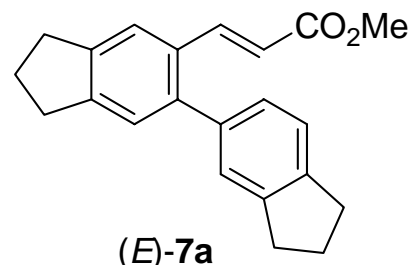

(96:4 E/Z)
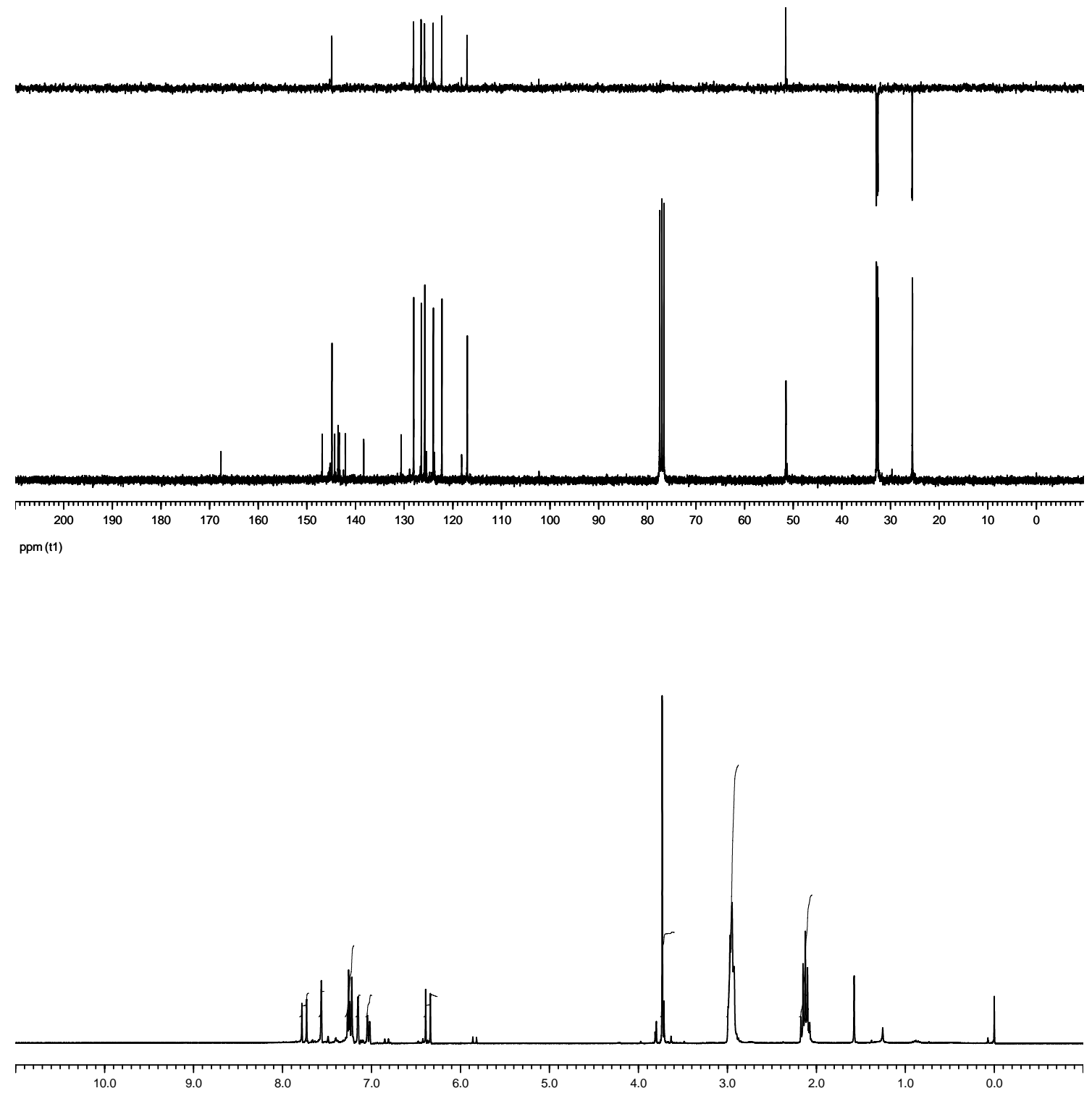

ppm (t1) 

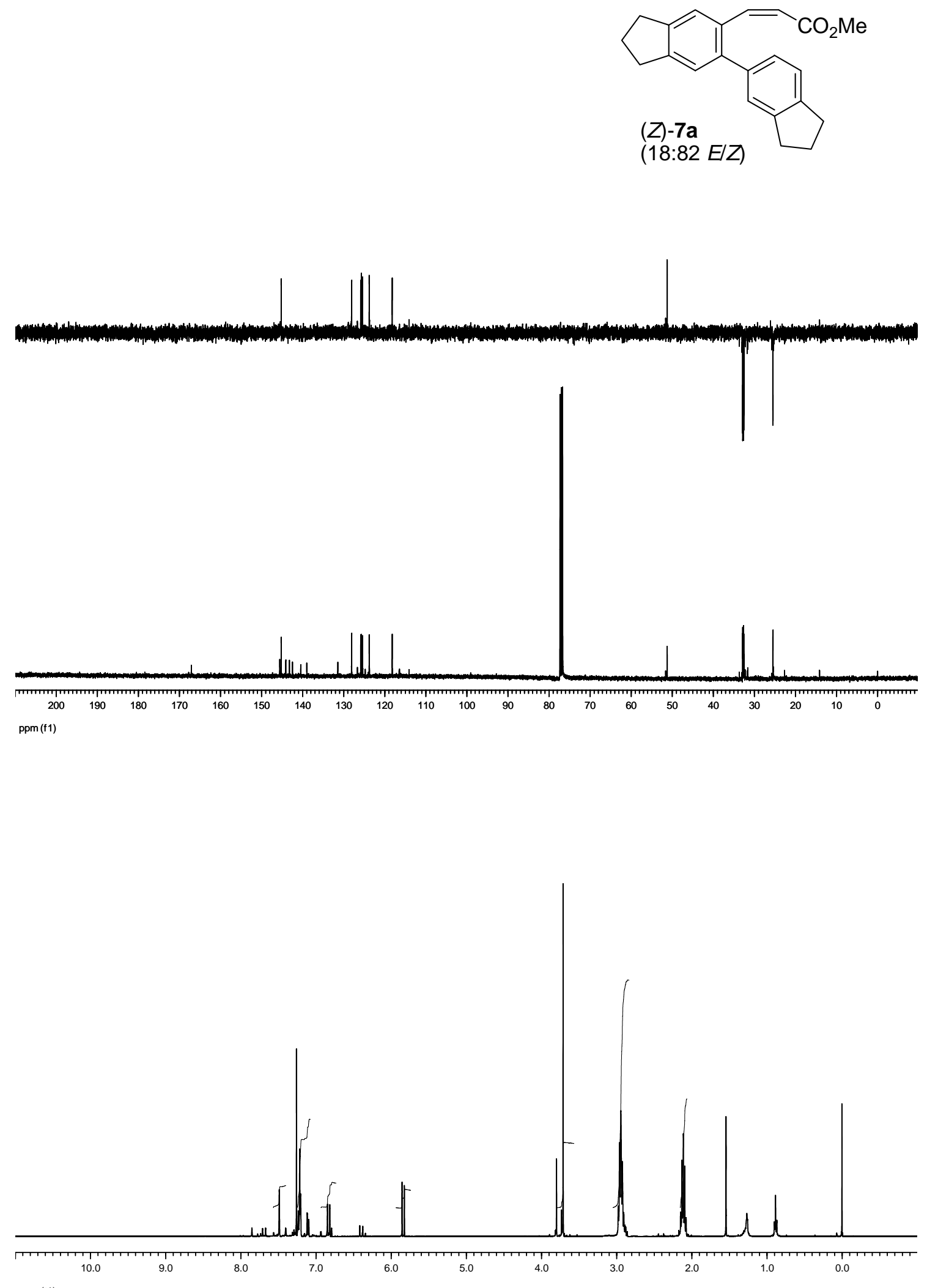

ppm (t1) 

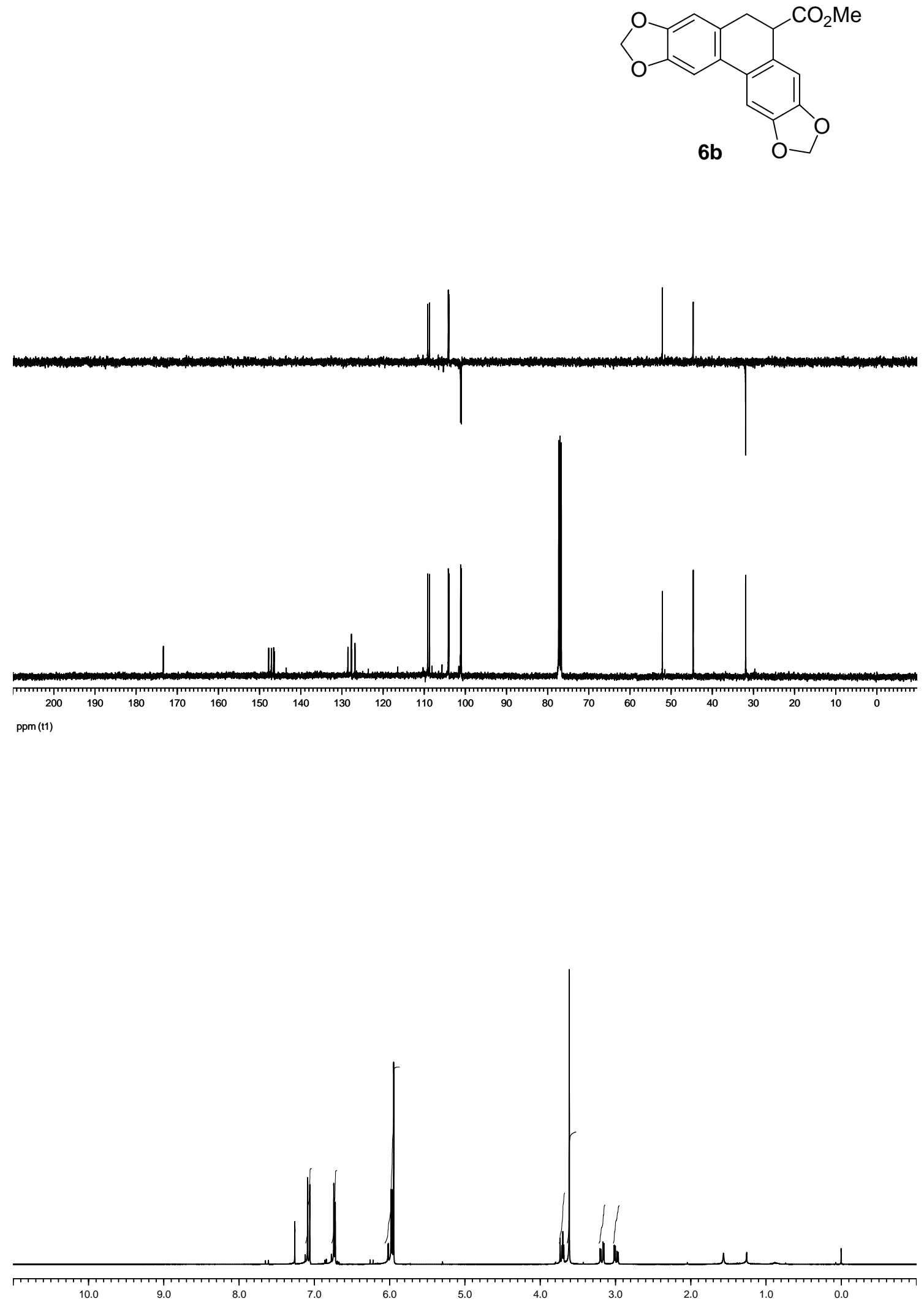

ppm (t1) 

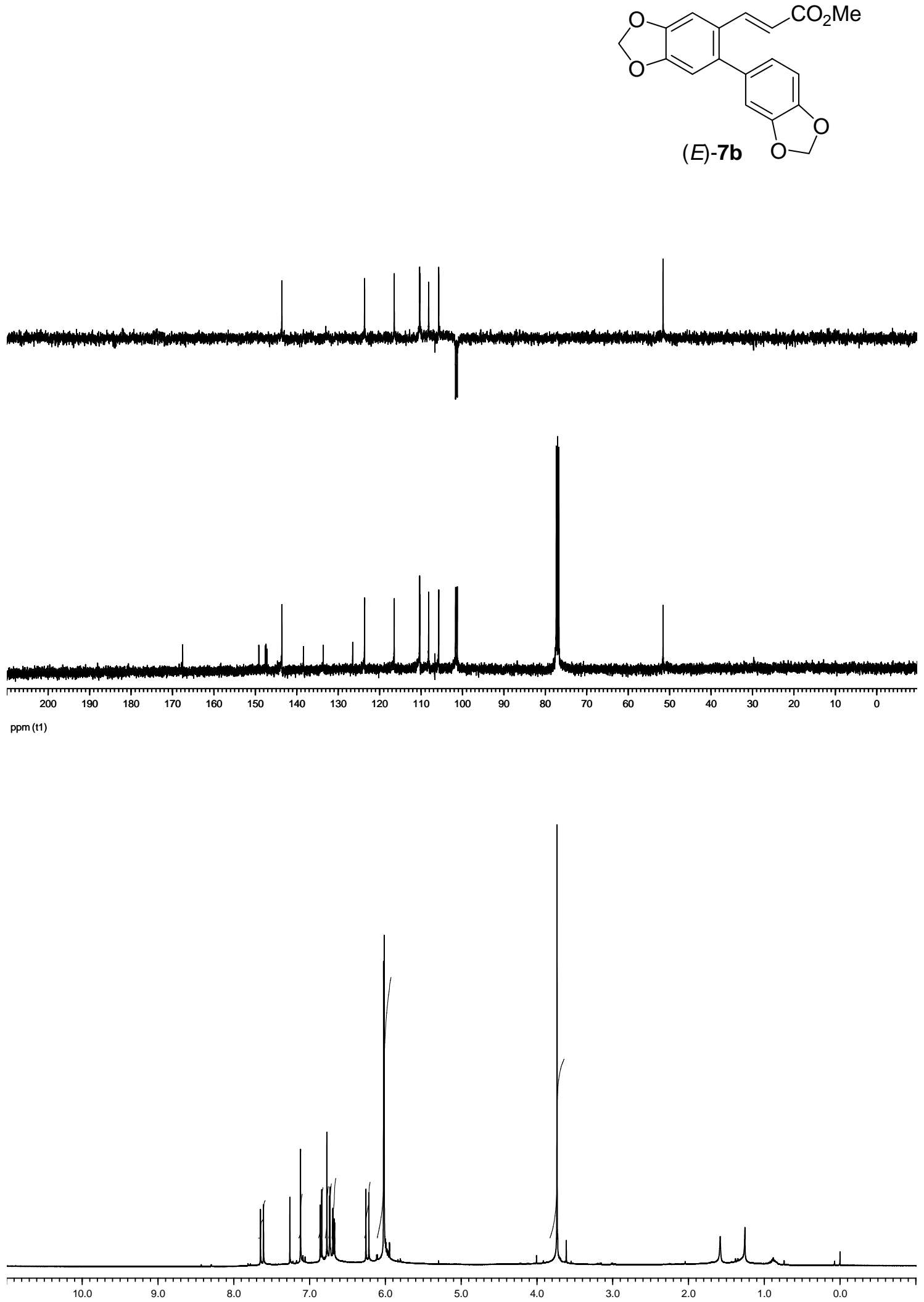

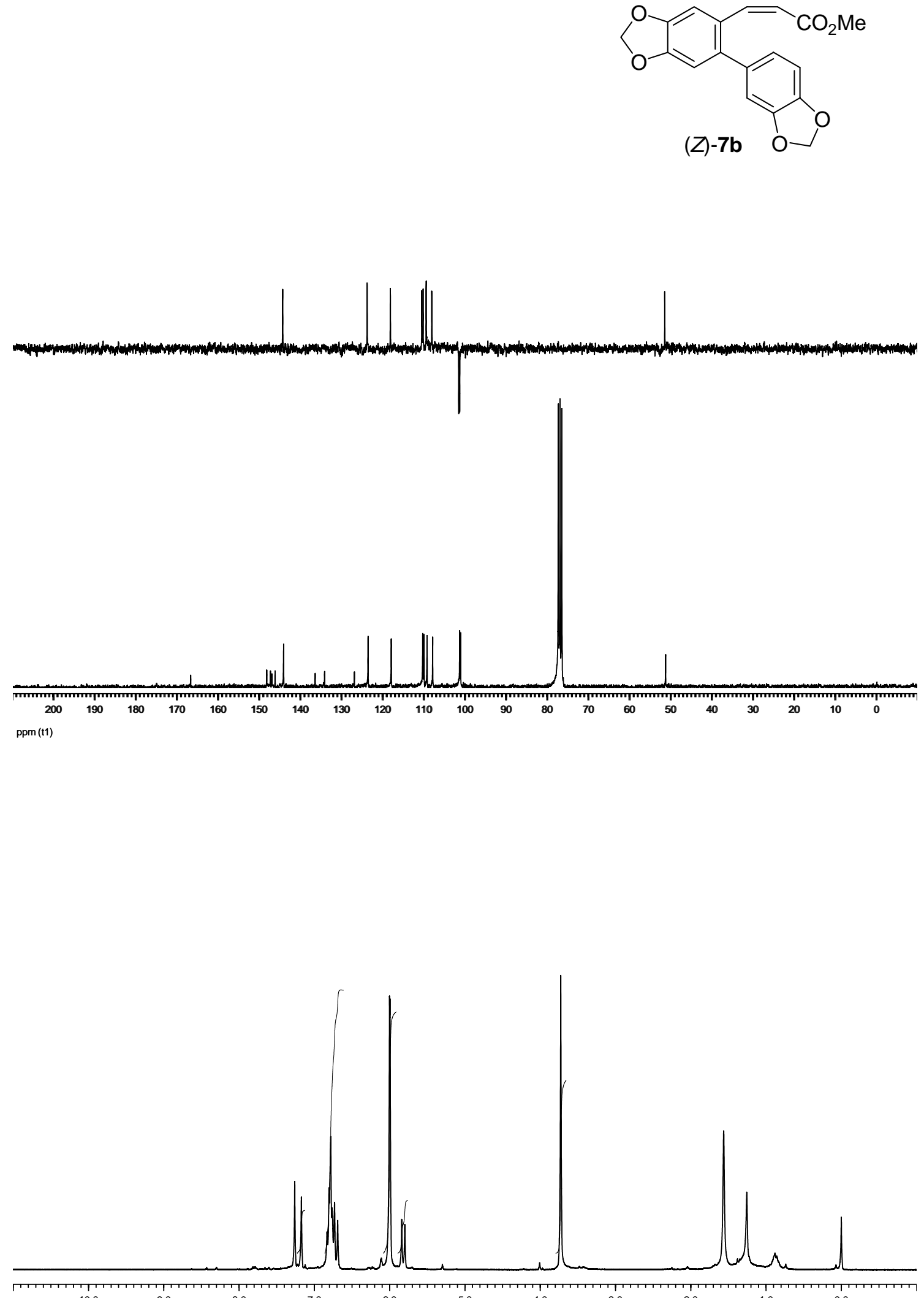
ppm (t1) 

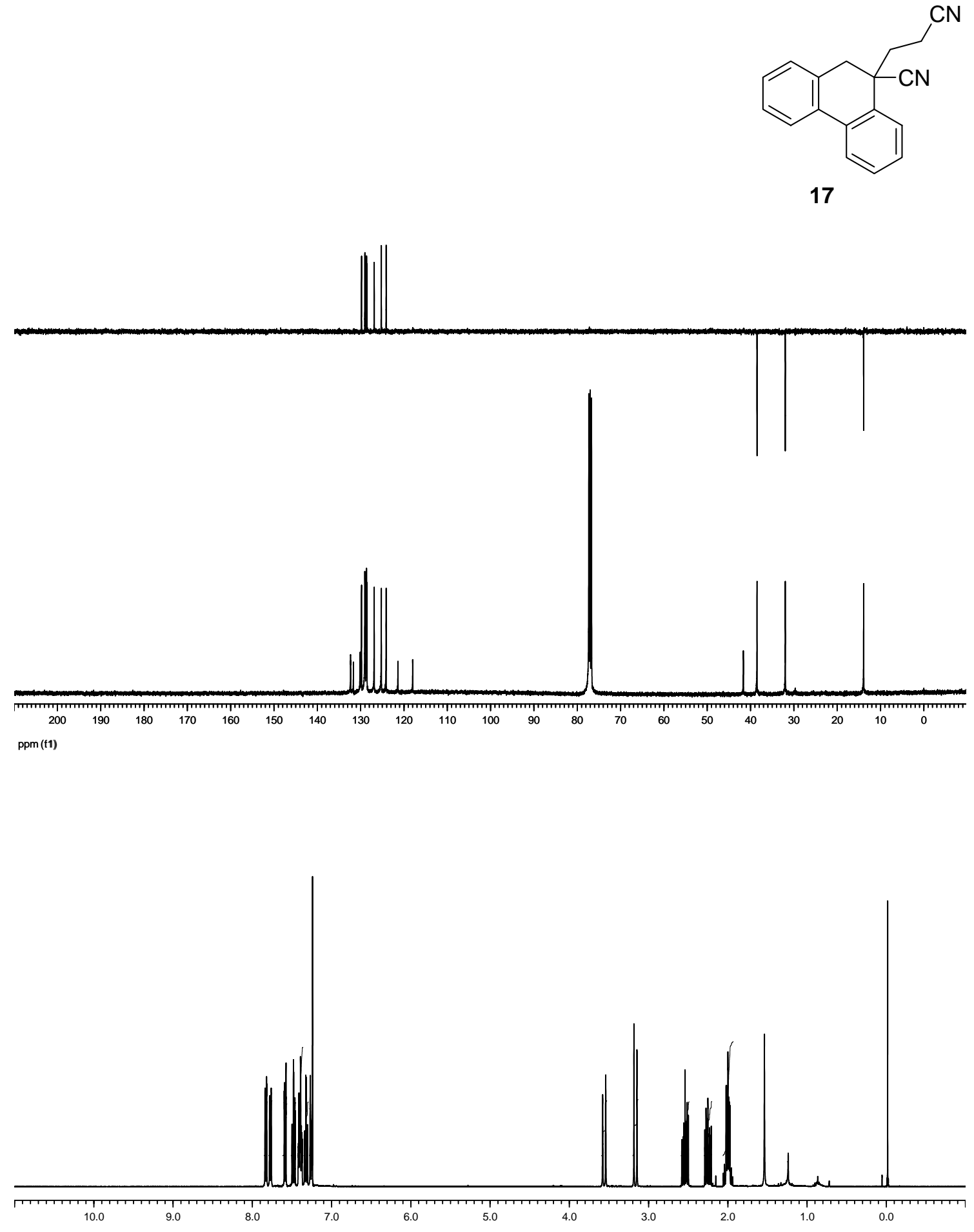

ppm (f1) 